\title{
Free boundaries touching the boundary of the domain for some reaction-diffusion problems
}

\author{
Jesus Ildefonso Díaz *, Tommaso Mingazzini ${ }^{\dagger}$
}

October 14, 2014

\section{Introduction}

We study the way the free boundary of solutions to some partial differential equations behaves depending on the trace of the solutions. The free boundary problems we consider are of two different types:

i) Elliptic reaction-diffusion type problems, as

$$
\begin{cases}-L u+\lambda u^{q}=0 & \text { in } \Omega, \\ u=h & \text { on } \partial \Omega,\end{cases}
$$

under the fundamental assumption

$$
q \in(0,1),
$$

which guaranties the formation of the free boundary (at least for $\lambda>0$ large enough, if $\Omega$ is bounded, or for any $\lambda>0$, if $\Omega$ is unbounded). Such problem arises, for instance, in Chemical Engineering when a catalytic chemical reactor occupying a domain $\Omega$ has a reactant feed channel (entrance boundary) which is represented by the part $\Gamma_{+} \subset \partial \Omega$, where the reactant concentration is $h(x)>0$ and the rest of walls of the chemical reactant are isolated in such a way that, if we denote by $\Gamma_{0}:=\partial \Omega \backslash \Gamma_{+}$, then $h(x)=0$ on $\Gamma_{0}$. Here we assume that there is no exit boundary (see Figure 1). The exponent $q$ is called the order of the reaction.

ii) The obstacle problem

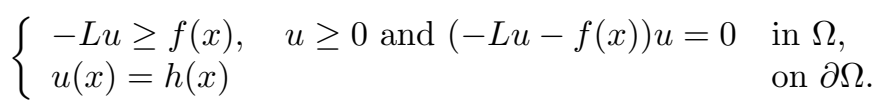

Here the free boundary is given by the boundary of the coincidence set (the set of points where $u=0$ ); according for instance to [17] a sufficient condition for the existence of the free boundary is that $f(x) \leq-\mu$ for some $\mu>0$ on a large enough open subset of $\Omega$ (see, for instance, 29] for a full treatment of the

\footnotetext{
*Instituto de Matemática Interdisciplinar, Universidad Complutense de Madrid, Plaza de las Ciencias 3, 28040 Madrid, España -

(ildefonso.diaz@mat.ucm.es).

†Universidad Complutense de Madrid, Dpto. de Matemática Aplicada, Plaza de las Ciencias 3, 28040 Madrid, España -

(tommaso.mingazzini@gmail.com).
} 


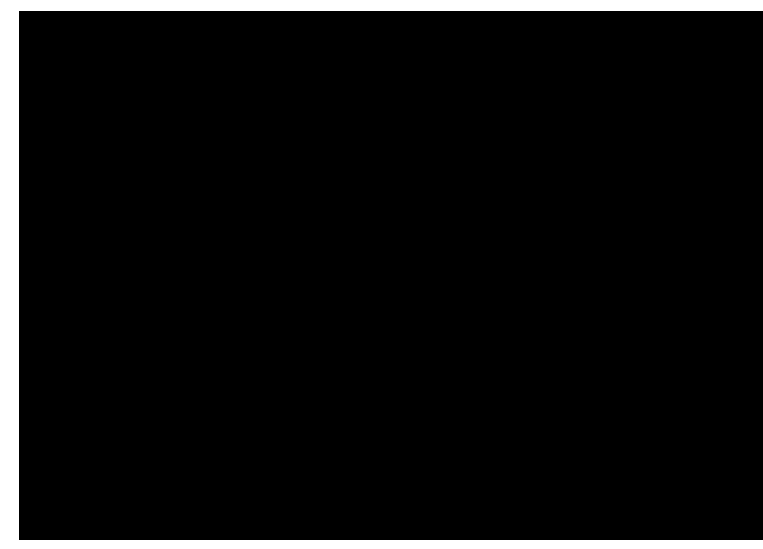

Figure 1: Chemical reactor scheme

obstacle problem). Among the many frameworks in which the obstacle problem arises we could mention, for instance, the unilateral problem of the stationary shape of a membrane which is forced downwards by a constant force $f$, is fixed on the boundary to a hight $h(x)$ and constrained to lie over the hyperplane $u=0$. Actually, here we shall consider the special case in which (3) can be formulated in terms of

$$
\begin{cases}-L u+\lambda \beta(u) \ni \varepsilon & \text { in } \Omega, \\ u=h & \text { on } \partial \Omega,\end{cases}
$$

for some constant $\varepsilon \in[0, \lambda)$, where $\beta(u)$ is the maximal monotone graph of $\mathbb{R}^{2}$ given by

$$
\beta(u)= \begin{cases}0 & \text { for } u<0, \\ {[0,1]} & \text { for } u=0, \\ 1 & \text { for } u>0 .\end{cases}
$$

If $u$ "solves" problem (4) (the rigorous definition of solution will be given later) then $u$ is also a solution of the obstacle problem (3) with $f=-\lambda+\varepsilon$ : indeed, we will see that $\varepsilon \geq 0$ and $h \geq 0$ imply that $u \geq 0$. Then, if $u>0,-L u+\lambda=\varepsilon$ which is the same as $-L u-f=0$. Finally, since there is uniqueness of solution for both formulations we get that the solutions must be the same.

Another interesting application of problem (4) arises also in the context of Chemical Engineering (as problem (1) with $q=0$ : see, e.g., [7]).

For some general purposes, such as the existence, uniqueness and regularity of the solutions, the domain $\Omega$ will be assumed to be an open regular set of $\mathbb{R}^{N}$. Nevertheless, when studying the qualitative properties of the solutions we focus on the bi-dimensional case, and we adopt as domain $\Omega$ both a bounded rectangle and the upper half plain in $\mathbb{R}^{2}$, i.e., $\Omega=\mathbb{R} \times[0, \infty)$. In the unbounded setting we use the following notation: $x:=\left(x_{1}, x_{2}\right)$ with $x_{1} \in \mathbb{R}$ and $x_{2} \in[0, \infty)$. The unbounded boundary of the domain is then $\partial \Omega=\mathbb{R} \times\{0\}$ and so the boundary function $h$ will depend only on the variable $x_{1}$. 
In general, $L$ denotes a second order elliptic operator of the form

$$
L u=\sum_{i, j=1}^{N} \frac{\partial}{\partial x_{i}}\left(a_{i j}(x) \frac{\partial}{\partial x_{j}} u\right)=\operatorname{div}(\mathbf{A}(\mathbf{x}) \nabla u),
$$

with $a_{i j} \in C^{1, \alpha}(\bar{\Omega})$ for some $\alpha \in(0,1)$, such that the corresponding matrix $\mathbf{A}(\mathbf{x})$ is symmetric and positive definite. Actually, in the parts concerning the behaviour of the support and free boundary of the solutions we shall restrict to the case of constant coefficients. This restriction serves merely to simplify the calculations and does not affect the local behaviour. For what concerns the boundary datum $h$, we assume that

$$
h \in L^{\infty}(\partial \Omega) \text { and } h \geq 0 \text { on } \partial \Omega,
$$

even though the existence and uniqueness results on a bounded domain hold for $h \in L^{1}(\partial \Omega)$ (and even for signed boundary measures).

A general exposition containing many references on both problems can be found in the monograph [17. One can see that both problems are special cases of the wider formulation

$$
\begin{cases}-L u+\lambda \beta(u) \ni f & \text { in } \Omega, \\ u=h & \text { on } \partial \Omega,\end{cases}
$$

where $\beta(u)$ is a maximal monotone graph of $\mathbb{R}^{2}$ such that $0 \in \beta(0)$ : $\beta$ is given by

$$
\beta(u)=|u|^{q-1} u
$$

in case of problem (1) and by (5) in case of problem (3). We define, as usual, the domain of $\beta$ as $D(\beta)=\{r \in \mathbb{R}: \beta(r) \neq \emptyset\}$ where $\emptyset$ stands for the empty set.

We also consider the associated parabolic problem

$$
\begin{cases}u_{t}-L u+\lambda \beta(u) \ni f(x, t) & \text { in } Q_{\infty}, \\ u=h(t, x) & \text { on } \Sigma_{\infty}, \\ u(x, 0)=u_{0}(x) & \text { on } \Omega,\end{cases}
$$

where $Q_{\infty}=\Omega \times(0, \infty), \Sigma_{\infty}=\partial \Omega \times(0, \infty)$ and for some $f \in L^{\infty}\left(Q_{\infty}\right) \cap$ $L_{\text {loc }}^{1}\left((0, \infty) ; L_{\text {loc }}^{1}(\Omega)\right), h \in L^{\infty}\left(\Sigma_{\infty}\right) \cap L_{\text {loc }}^{1}\left((0,+\infty) ; L^{1}(\partial \Omega)\right)$, with $f, h \geq 0$ respectively on $Q_{\infty}$ and on $\Sigma_{\infty}$, and $u_{0} \in L^{\infty}(\Omega)$ with $u_{0} \geq 0$ on $\Omega$.

As mentioned before, the above problems, both elliptic and parabolic, give rise to a free boundary defined as the boundary of the support of the solution. If we denote the positivity set of a non-negative function $u$ by $\mathcal{S}(u):=$ $\{x \in \Omega: u(x)>0\}$, then the free boundary is defined as $\mathcal{F}(u)=\partial \mathcal{S} \cap \Omega$ (we also introduce the null set of $u$ as $\mathcal{N}(u):=\{x \in \Omega: u(x)=0\}$ and the support of $u$ as $\overline{\mathcal{S}}(u))$. Similar notations can be introduced also for the parabolic problem, applying the definitions to $u(t, \cdot)$.

Our main goal in this work is to study the behaviour of the free boundary near the support of the boundary datum $h$ (respectively $h(t, \cdot)$ ). For this purpose, we shall assume that

$$
\mathcal{S}(h) \subsetneq \partial \Omega,
$$


respectively

$$
\mathcal{S}(h(t, \cdot)) \subsetneq \partial \Omega \text {, for a.e. } t>0 .
$$

The main question we investigate is whether the free boundary $\mathcal{F}(u)$ is connected or not with the boundary of the support of the boundary datum $h$ (and similar question for the parabolic formulation). In some sense, this research can be considered as a natural continuation of the study of the so called non-diffusion of the support property (see [17] and [2]) in the case where $h \equiv 0$; under a suitable behaviour of $f$ near the boundary of its support $\mathcal{S}(u)=\mathcal{S}(f)$. In the case of parabolic free boundary problems this question is related with the behaviour of the free boundary for small times (the so called waiting time property) and received a great attention in the last 40 years (see, e.g., the monographs 31, 3. and their many references). Another study, not too far from our interest is the paper by Martel and Souplet 27] regarding the behaviour of solutions of linear parabolic problems with incompatible initial data.

To be more precise, our main goal is to find some sufficient criterion on the behaviour of $h$ near the boundary of its support ensuring that the free boundary $\mathcal{F}(u)$ is in contact with $\partial \mathcal{S}(h)$. In this way the support of the datum is not diffused on the boundary of the domain and we would have

$$
\partial \mathcal{S}(u) \cap \partial \Omega=\mathcal{S}(h)
$$

It is what we can call the non-diffusion on the boundary of the support property. In addition, we want to give some sufficient conditions ensuring the opposite qualitative behaviour, i.e., to find conditions on $h$ implying that there is a strict expansion of the support $\mathcal{S}(h)$ on the boundary $\partial \Omega$. In other words, we want to know cases in which $\mathcal{F}(u)$ has no contact with $\partial \mathcal{S}(h)$ and so

$$
\mathcal{S}(h) \subsetneq \partial \mathcal{S}(u) \cap \partial \Omega
$$

We call this phenomenon the expansion on the boundary of the support property. The only paper in the previous literature about such boundary qualitative behaviour we are aware of is [20] in which they proved the expansion on the boundary of the support property for problem (1) in the special case of $L u=\Delta u$, $h$ given by the Heaviside function and $\Omega$ the half plane $\mathbb{R} \times \mathbb{R}^{+}$. As we shall see later, this property holds even for suitable continuous boundary data $h$.

We point out that there is an extensive literature dealing with the regularity of the free boundary when it touches the fixed boundary for the special case of the obstacle problem (both in the elliptic and parabolic case): see, e.g. 4, [5], 6] and [30. In some of those papers the authors also consider more general diffusion operators (arriving to consider even the case of fully nonlinear second order operators). Nevertheless, the reaction term here involved, $\beta(u)$, is never similar to the one considered in those papers except for the case of zero-order reaction. In any case, our main interest is not the regularity of the free boundary but its qualitative behaviour depending on the data of the problem (specially the behaviour of the boundary datum near its support). To stretch the value of our results, we underline that the techniques of proof in this paper are quite different to the ones used in the above mentioned papers on the obstacle problem.

Before stating our main results we need to make precise the notion of solution. The delicate point in our study is that we want to allow the boundary 
datum to be discontinuous and so the notion of the trace of the solution must be taken in a very general framework (something which, in our opinion, is not discussed enough in [20]).

We recall that the notion of boundary trace of a function $u$ in $\Omega$ depends on the regularity properties of such function $u$. For instance, when $u \in C(\bar{\Omega})$ the boundary trace $\left.u\right|_{\partial \Omega}$ is clearly well defined and belongs to $C(\partial \Omega)$. If $u$ is in some Sobolev space $W^{1, p}(\Omega)$, for some $p>1$, then the boundary trace can be defined and is a function in the space $L^{p}(\partial \Omega)$ (more precisely in the Sobolev space $W^{1-\frac{1}{p}, p}(\partial \Omega)$ : see, e.g., [23] and [1]) . Nevertheless, the identification of the elements of the trace space $W^{1-\frac{1}{p}, p}(\partial \Omega)$ is not always easy and leads to some pathological results against intuition. For instance in the book by Mikhailov [28] one can see that already when $\Omega=B$, the unit ball of $\mathbb{R}^{2}$, there are continuous functions $h \in C(\partial B)$ which are not the trace of any function in $H^{1}(\Omega)$ (i.e., $\left.C(\partial B) \nsubseteq H^{\frac{1}{2}}(\partial B)\right)$.

A different approach was proposed by Haïm Brezis, in an unpublished paper (1972) profusingly mentioned in the literature (see [32, 26] and 19]), which holds for semilinear second order boundary value problems with boundary data in $L^{1}(\partial \Omega)$ (later extended to measures on $\partial \Omega$ ). The main idea is to multiply by a "regular" test function $\left(\varphi \in W^{2, \infty}(\Omega) \cap W_{0}^{1, \infty}(\Omega)\right)$ and to integrate twice by parts. We introduce the adjoint operator

$$
L^{*} u=\sum_{i, j=1}^{N} \frac{\partial}{\partial x_{j}}\left(a_{i j}(x) \frac{\partial}{\partial x_{i}} u\right)=\operatorname{div}\left(\mathbf{A}^{*} \nabla u\right)
$$

( $\mathbf{A}^{*}$ the transposed matrix of $\mathbf{A}$ ) and for $x \in \partial \Omega$ we define

$$
\partial_{A} u:=\left(A^{*} \nabla u\right) \cdot \mathbf{n},
$$

where $\mathbf{n}(x)$ is the outward normal vector to $\partial \Omega$ in $x$. A solution is then a function $u$ which satisfies

$$
-\int_{\Omega} u L^{*} \varphi \mathrm{d} x+\lambda \int_{\Omega} b \varphi \mathrm{d} x=\int_{\Omega} f \varphi \mathrm{d} x-\int_{\partial \Omega} h \partial_{A} \varphi \mathrm{d} \sigma
$$

for all $\varphi \in W^{2, \infty}(\Omega) \cap W_{0}^{1, \infty}(\Omega)$ and for some $b \in L_{l o c}^{1}(\Omega)$ such that $b(x) \in$ $\beta\left(u(x)\right.$ ) for a.e. $x \in \Omega$ (in case of problem (1), it is $b=u^{q}$ ). In order to give a meaning to all the above integrals, it is useful to recall that, since $\varphi \in W_{0}^{1, \infty}(\Omega)$, it holds $c_{1} \rho(x) \leqslant \varphi(x) \leqslant c_{2} \rho(x) \forall x \in \Omega$, where

$$
\rho(x):=\operatorname{dist}(x, \partial \Omega)
$$

and $c_{1}, c_{2}$ are positive constants. Thus we must require at least that

$$
f \in L^{1}(\Omega ; \rho),
$$

where

$$
L^{1}(\Omega ; \rho):=\left\{f \in L_{l o c}^{1}(\Omega): \int_{\Omega}|f(x)| \rho(x) \mathrm{d} x<+\infty\right\}
$$




\section{$1.1 \quad$ Elliptic case}

Definition 1.1. Given $f \in L^{1}(\Omega ; \rho)$ and $h \in L^{1}(\partial \Omega)$, we say that $u$ is a very weak solution of problem (7) if $u \in L^{1}(\Omega)$ and there exists $b \in L^{1}(\Omega ; \rho)$ such that $b(x) \in \beta\left(u(x)\right.$ ) for a.e. $x \in \Omega$, and for any test function $\varphi \in W^{2, \infty}(\Omega) \cap$ $W_{0}^{1, \infty}(\Omega)$ identity $(11)$ holds.

It is not too difficult to adapt to our framework some existence and uniqueness results in the literature (see [12], 32] and [26]).

Theorem 1.2. Let $\Omega$ be a bounded regular open set of $R^{N}$, let $\beta$ be a maximal monotone graph of $R^{2}$ such that $0 \in \beta(0)$ and let $f \in L^{1}(\Omega ; \rho)$ and $h \in L^{1}(\partial \Omega)$. Then there exists a unique very weak solution u of problem (7). Moreover, there exists a constant $C$, only dependent of $\Omega$, such that if $\widehat{u}$ is the very weak solution corresponding to the data $\widehat{f} \in L^{1}(\Omega ; \rho)$ and $\widehat{h} \in L^{1}(\partial \Omega)$, with $\widehat{b}(x) \in \beta(\widehat{u}(x))$ for a.e. $x \in \Omega$ as in Definition 1.1, then we have

$$
\begin{aligned}
\left\|[u-\widehat{u}]_{+}\right\|_{L^{1}(\Omega)}+\lambda\left\|[b-\widehat{b}]_{+}\right\|_{L^{1}(\Omega ; \rho)} & \\
\leq C & \left(\left\|[f-\widehat{f}]_{+}\right\|_{L^{1}(\Omega ; \rho)}+\left\|[h-\widehat{h}]_{+}\right\|_{L^{1}(\partial \Omega)}\right)
\end{aligned}
$$

and

$$
\begin{aligned}
\|u-\widehat{u}\|_{L^{1}(\Omega)} & +\lambda\|b-\widehat{b}\|_{L^{1}(\Omega ; \rho)} \\
& \leq C\left(\|f-\widehat{f}\|_{L^{1}(\Omega ; \rho)}+\|h-\widehat{h}\|_{L^{1}(\partial \Omega)}\right) .
\end{aligned}
$$

In particular, $f \leq \widehat{f}$ and $h \leq \widehat{h}$ imply that $u \leq \widehat{u}$ on $\Omega$.

To study the behaviour of the solution close to the boundary of $\mathcal{S}(h)$ we consider two different frames, an unbounded case and a bounded one.

In the first case we set $\Omega=\mathbb{R} \times[0, \infty), a_{i j}$ is constant for $i, j \in\{1,2\}$, $f \equiv 0$, and $\beta(u)$ is given by (8) or (5). For what concerns the boundary datum, we are interested in the case of $h$ satisfying, $h \in L^{\infty}(\partial \Omega), h\left(x_{1}\right)=$ 0 on $(-\infty, 0)$ and $h\left(x_{1}\right)>0$ on $(0,+\infty)$.

The reason why we consider boundary data in $L^{\infty}(\partial \Omega)$ instead of in $L^{1}(\partial \Omega)$ (remember that now $\partial \Omega$ is unbounded, so $L^{\infty}(\partial \Omega) \nsubseteq L^{1}(\partial \Omega)$ ) is that we know the explicit solution in the unperturbed linear case $(\lambda=0, L=\Delta, f \equiv 0)$ with boundary data given by the Heaviside function $(52)$. Such solution is given by

$$
u\left(x_{1}, x_{2}\right)=1-\frac{1}{\pi} \arctan \left(\frac{x_{1}}{x_{2}}\right)
$$

(the result can be found in [20] formula (2.6)). Having at disposal an explicit solution like (14) is really useful in the study of the behaviour of general solutions close to the point $x=(0,0)$. In addition, since our main interest, as already said, is specifically the behaviour near the boundary of the support $\partial \mathcal{S}(h)$ and not in the whole $\Omega$, we shall assume also that $h$ is non decreasing and that 
$h\left(x_{1}\right)=c_{+}>0$ for $x_{1} \geq \delta>0$. We can resume this set of hypothesis in

$$
\left\{\begin{array}{l}
\Omega=\mathbb{R} \times[0, \infty), a_{i j} \text { constant for } i, j \in\{1,2\}, f \equiv 0, \\
\beta(u) \text { is given by (8) or }(5), \\
h \in L^{\infty}(\partial \Omega), h\left(x_{1}\right)=0 \text { on }(-\infty, 0), \\
h\left(x_{1}\right)>0 \text { on }(0, \infty) \text { and } h\left(x_{1}\right)=c_{+}>0 \text { on }(\delta,+\infty) .
\end{array}\right.
$$

To give a definition of solution for the new setting $H_{h p}$, we first introduce the family of rectangles

$$
R_{n}=\left\{\left(x_{1}, x_{2}\right):\left|x_{1}\right|<n, 0<x_{2}<\bar{x}\right\},
$$

where $\bar{x}$ is a constant which will be make explicit later on. We define now the bounded domain $\Omega_{n}$, which is a sufficiently smooth regularization of the rectangular $R_{n}$. We call $\Gamma_{n}^{1}$ the horizontal boundary of $\Omega_{n}$, i.e., $\Gamma_{n}^{1}:=\left\{\left(x_{1}, x_{2}\right) \in\right.$ $\partial \Omega_{n}: x_{2}=0$ or $\left.x_{2}=\bar{x}\right\}$, and $\Gamma_{n}^{2}=\partial \Omega_{n} \backslash \Gamma_{n}^{1}$. Then we consider the problem

$$
\left\{\begin{array}{lll}
-L u+\beta(u) \ni 0 & \text { in } \Omega_{n}, \\
u\left(x_{1}, 0\right)=h\left(x_{1}\right), & u\left(x_{1}, \bar{x}\right)=0, & \left|x_{1}\right| \leq n, \\
u\left(x_{1}, x_{2}\right)=0 & \left(x_{1}, x_{2}\right) \in \Gamma_{n}^{2}, & x_{1}<0, \\
u\left(x_{1}, x_{2}\right)=z\left(x_{2}\right) & \left(x_{1}, x_{2}\right) \in \Gamma_{n}^{2}, & x_{1}>0,
\end{array}\right.
$$

where $z$ is given by (51). We define the class of "limit very weak solutions".

Definition 1.3. In the framework of $H_{h p}$, we say that $u$ is a limit very weak solution of problem (7) if $u=\lim _{n \rightarrow \infty} u_{n}$, where $u_{n}$ is the solution of the truncated problem (15).

Theorem 1.4. Assume $\left(H_{h p}\right)$. There exists a unique limit very weak solution of problem (7) on the hyperplane $\Omega=\mathbb{R} \times[0, \infty)$. Moreover that solution satisfies the comparison principle with respect to the boundary data $h$ : if $h \leq \widehat{h}$ then the corresponding limit very weak solutions satisfy $u \leq \widehat{u}$ on $\Omega$.

Our main result concerns the qualitative behaviour of the solution of (7) under the assumption $H_{h p}$.

Theorem 1.5. Assume $\left(H_{h p}\right)$. Then there exist four positive constants $\underline{C}<\bar{C}$, $\underline{\varepsilon}<\bar{\varepsilon}$ and two boundary points $\underline{x}_{1, \varepsilon}, \bar{x}_{1, \varepsilon}>0$, such that :

i) If $h\left(x_{1}\right) \geq \bar{C} x_{1}^{\frac{2}{1-q}}$ for a.e. $x_{1} \in\left(0, \bar{x}_{1, \varepsilon}\right)$ and $h(x) \geq \bar{\varepsilon}$ for a.e. $x_{1} \in$ $\left(\bar{x}_{1, \varepsilon},+\infty\right)$, then the expansion on the boundary of the support property holds.

ii) If $h\left(x_{1}\right) \leq \underline{C} x_{1}^{\frac{2}{1-q}}$ for a.e. $x_{1} \in\left(0, \underline{x}_{1, \varepsilon}\right)$ and $h\left(x_{1}\right) \leq \underline{\varepsilon}$ for a.e. $x_{1} \in$ $\left(\underline{x}_{1, \varepsilon},+\infty\right)$, then the non-diffusion on the boundary of the support property holds. In both cases, $q \in(0,1)$ when $\beta$ is given by (8) and $q=0$ when $\beta$ is chosen as (5).

The indicative qualitative behaviour of solutions illustrated in Theorem 1.5 is resumed in Figure 2 ( $i$ ) and 3 ( $i i$.

Corollary 1.6. In the same framework of Theorem 1.5 we have the additional consequences: 


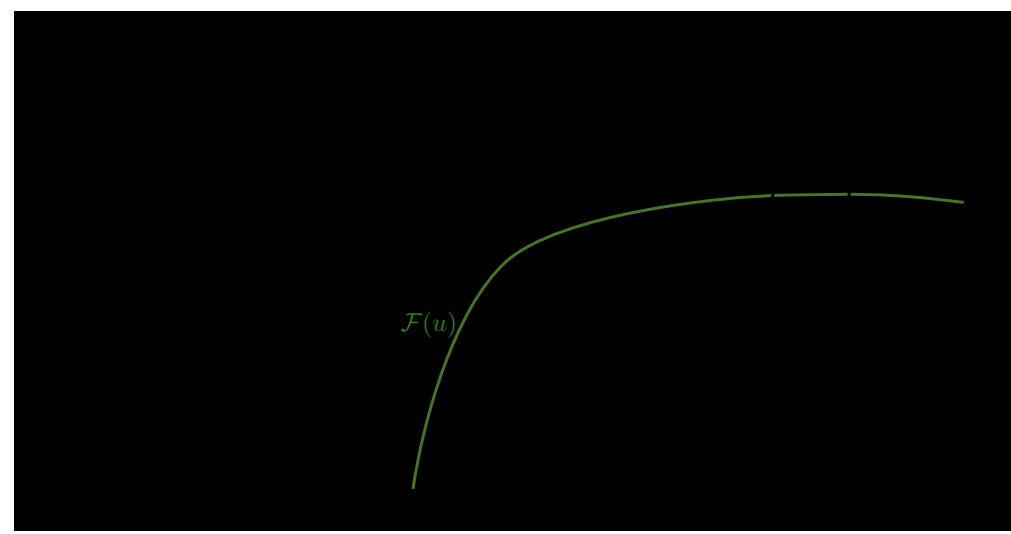

Figure 2: Expansion on the boundary of the support

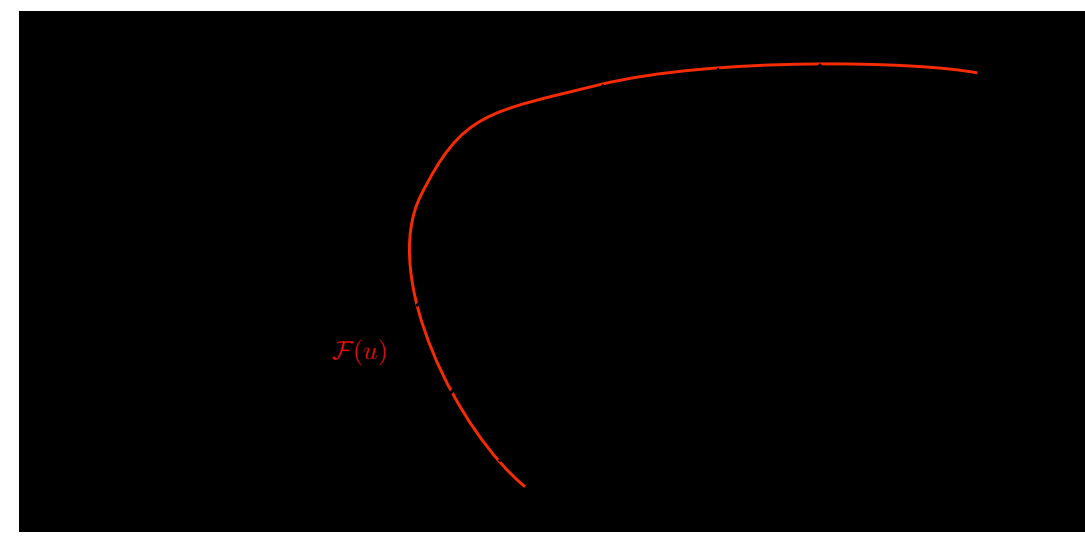

Figure 3: Non-diffusion on the boundary of the support 
1) Under condition (i), $u=\frac{\partial}{\partial n} u=0$ on $(-\infty, 0) \times\{0\}$.

2) Under condition (ii), $\frac{\partial}{\partial n} u>0$ on $(-\zeta, 0) \times\{0\}$ for some $\zeta>0$. If in particular $h$ is a multiple of the Heaviside function, then $\frac{\partial}{\partial n} u \notin C(\partial \Omega)$ and $u \notin C(\bar{\Omega})$.

In both situations, $n=(0,1)$ is the normal vector to $\partial \Omega$.

For the bounded case, we set $\Omega \subset \mathbb{R}^{2}$ to be the rectangle of vertexes

$$
R=\left\{\left(-B_{l}, 0\right),\left(B_{r}, 0\right),\left(B_{r}, B_{u}\right),\left(-B_{l}, B_{u}\right)\right\} \text { with } B_{l}, B_{r}, B_{u}>0 .
$$

Let us call

$$
\begin{array}{llrl}
\partial_{1} R & =\left[\left(-B_{l}, 0\right),\left(B_{r}, 0\right)\right], & \partial_{2} R=\left[\left(B_{r}, 0\right),\left(B_{r}, B_{u}\right)\right], \\
\partial_{3} R=\left[\left(B_{r}, B_{u}\right),\left(-B_{l}, B_{u}\right)\right], & \partial_{4} R=\left[\left(-B_{l}, B_{u}\right),\left(-B_{l}, 0\right)\right],
\end{array}
$$

the four sides of $R$. The internal datum $f$ is set to zero. We have the following result.

Theorem 1.7. Assume that

$$
h= \begin{cases}\varepsilon H\left(x_{1}\right) & \text { on } \partial_{1} R, \\ \max \left(0, \psi\left(x_{2}\right)\right) & \text { on } \partial_{2} R, \\ 0 & \text { on } \partial_{3} R \cup \partial_{4} R,\end{cases}
$$

with $H\left(x_{1}\right)=0$ for $x_{1}<0, H\left(x_{1}\right)=1$ for $x_{1} \geq 0$ and

$$
\psi\left(x_{2}\right)=\varepsilon\left(\frac{1}{2}+\frac{1}{\pi} \arctan \left((\operatorname{det} A)^{-1}\left(a_{22} \frac{B_{r}}{x_{2}}-a_{12}\right)\right)\right)+\frac{\lambda \varepsilon^{q}}{2} x_{2}^{2}+C x_{2}
$$

where $C$ is a negative constant. If

$$
C \leq \min \left(-\frac{\varepsilon}{B_{u}}-\frac{\lambda \varepsilon^{q}}{2} B_{u},-\frac{\varepsilon(\operatorname{det} A)^{1 / 2}}{\pi a_{22} B_{l}}\right),
$$

the the solution of (7) with boundary datum $h$ and internal datum $f \equiv 0$ satisfies the expansion on the boundary of the support property. In both cases, $q \in(0,1)$ when $\beta$ is given by (8) and $q=0$ when $\beta$ is chosen as (5).

Proof. First of all we want to point out that since the boundary conditions are all non-negative and bounded by $\varepsilon$, then $0 \leq u \leq \varepsilon$.

We consider the subsolution given by $\underline{u}=u_{1}+u_{2}$, where

$$
\begin{array}{r}
u_{1}=\varepsilon\left(\frac{1}{2}+\frac{1}{\pi} \arctan \left((\operatorname{det} A)^{-1 / 2}\left(a_{22} \frac{x_{1}}{x_{2}}-a_{12}\right)\right)\right), \\
u_{2}=\frac{\lambda \varepsilon^{q}}{2} x_{2}^{2}+C x_{2} .
\end{array}
$$

One can easily check that $L u_{1}=0$ and $L u_{2}=\lambda \varepsilon^{q}$ and as a consequence

$$
-L u=-\lambda u^{q} \geq-\lambda \varepsilon^{q}=-L \underline{u} .
$$


Now let us check the boundary conditions for $\underline{u}$. On $\partial_{1} R$ we have that $\underline{u}\left(x_{1}\right)=$ $\varepsilon H\left(x_{1}\right)$. On $\partial_{2} R$ it is exactly $\psi\left(x_{2}\right)$. On $\partial_{3} R$, thanks to the hypothesis on $C$, it holds

$$
\underline{u}\left(x_{1}, B_{u}\right) \leq \varepsilon+\frac{\lambda \varepsilon^{q}}{2} B_{u}^{2}+C B_{u} \leq 0 .
$$

On $\partial_{4} R, \underline{u}\left(-B_{l}, 0\right)=0$. We compute the $x_{2}$-derivative

$$
\frac{\partial \underline{u}}{\partial x_{2}}\left(x_{1}, x_{2}\right)=\lambda \varepsilon^{q} x_{2}+C-\frac{\epsilon}{\pi} \frac{a_{22}(\operatorname{det} A)^{1 / 2} x_{1}}{\operatorname{det} A x_{2}^{2}+\left(a_{22} x_{1}-a_{12} x_{2}\right)^{2}}
$$

With a direct computation and thanks to the condition on $C, \partial \underline{u} / \partial x_{2}\left(-B_{l}, 0\right)<$ 0 and $\partial \underline{u} / \partial x_{2}\left(-B_{l}, x_{1}\right)$ is an increasing function. Hence, since $\underline{u}\left(-B_{l}, B_{u}\right) \leq 0$, $\underline{u}_{\mid \partial_{4} R} \leq 0$. We have proved that $\underline{u} \leq u$.

Now let us check that the expansion on the boundary of the support holds for $\underline{u}$ and as a consequence for $u$. We know that $\underline{u}\left(x_{1}, 0\right)=0$ for $x_{1}<0$. The derivative

$$
\frac{\partial \underline{u}}{\partial x_{2}}\left(x_{1}, 0\right)=C-\frac{\epsilon}{\pi} \frac{\operatorname{det} A}{a_{22} x_{1}}<0
$$

for $x_{1}>\frac{\epsilon \operatorname{det} A}{\pi a_{22} C}=-\eta$. It follows that $u>0$ in a neighbourhood in $\mathbb{R}^{2}$ of $(-\eta, 0) \times\{0\}$.

Corollary 1.8. The same result holds under the same conditions of the previous theorem but with $u_{\mid \partial_{2} R} \geq \psi$.

\subsection{Parabolic case}

As far as the parabolic problem is concerned, our main interest consists in analysing the stabilization of the solution to the solution of stationary problem in order to well understand the expansion on the boundary of the support property. When $\Omega$ is a general open bounded set the notion of very weak solution is quite similar to the elliptic case.

Definition 1.9. Take $T>0, f \in L^{1}\left(0, T ; L^{1}(\Omega ; \rho)\right), h \in L^{1}\left(0, T ; L^{1}(\partial \Omega)\right)$ and $u_{0} \in L^{1}(\Omega ; \rho)$ with $u_{0}(x) \in \overline{D(\beta)}$. We say that $u$ is a very weak solution of problem (9) if $u \in L^{1}\left(0, T ; L^{1}(\Omega)\right)$ and there exists $b \in L^{1}\left(0, T ; L^{1}(\Omega ; \rho)\right)$ such that $b(t, x) \in \beta(u(t, x))$ for a.e. $(t, x) \in(0, T) \times \Omega$, and for every test function $\varphi \in W^{1, \infty}\left([0, T] ; L^{\infty}(\Omega)\right) \cap L^{\infty}\left(0, T ; W^{2, \infty}(\Omega) \cap W_{0}^{1, \infty}(\Omega)\right)$ with $\varphi(T, \cdot)=0$ the following identity holds

$$
\begin{aligned}
-\int_{0}^{T} & \int_{\Omega} u \frac{\partial \varphi}{\partial t} \mathrm{~d} x \mathrm{~d} t+\int_{0}^{T} \int_{\Omega} u L^{*} \varphi \mathrm{d} x+\lambda \int_{0}^{T} \int_{\Omega} b \varphi \mathrm{d} x \mathrm{~d} t \\
= & \int_{\Omega} u_{0}(x) \varphi(0, x) \mathrm{d} x+\int_{0}^{T} \int_{\Omega} f \varphi \mathrm{d} x \mathrm{~d} t-\int_{0}^{T} \int_{\partial \Omega} h \partial_{A} \varphi \mathrm{d} \sigma \mathrm{d} t .
\end{aligned}
$$

Once again, it is not too difficult to adapt to our framework some existence and uniqueness results in the literature (see 24]).

Theorem 1.10. i) For data $f, h$ and $u_{0}$ as in Definition 1.9 there exists a unique very weak solution of (9). Moreover there holds a smoothing effect (same as [24]).

ii) If in addition $h \in W^{1,1}\left(0, T ; L^{1}(\partial \Omega)\right)$, then the very weak solution satisfies $u \in C\left([0, T] ; L^{1}(\Omega ; \rho)\right)$. 




Figure 4: Convergence of $\mathcal{S}(u(t, \cdot))$ to $\mathcal{S}\left(u_{\infty}\right)$

Our result on the asymptotic behaviour, for $t \rightarrow+\infty$, seems to be new in the context of very weak solutions (check [18] and 22] for similar results on more regular solutions).

Remark 1.11. Whenever we are dealing at the same time with the parabolic and the elliptic problem, as we are going to do, we use the symbols $u_{\infty}, h_{\infty}$, $f_{\infty}$ to denote the solution and the data of the elliptic boundary value problem.

For the next result we will add the following hypothesis:

$$
\begin{aligned}
& \exists q \in[0,1) \text { such that }|b| \leq C|r|^{q} \\
& \text { for any } b \in \beta(r) \text { and for any } r \in \mathbb{R} .
\end{aligned}
$$

In the above condition the case $q=0$ means that $R(\beta)$ (the range of $\beta$, i.e., $r \in \mathbb{R}$ such that there exists $x \in \mathbb{R}$ for which $r \in \beta(x))$ is bounded.

Theorem 1.12. Consider the case of $\beta$ satisfying condition (19). Assume $h \in$ $W^{1,1}\left(0, T ; L^{1}(\partial \Omega)\right)$ for any $T>0$ and that there exists a sequence $t_{n} \rightarrow+\infty$, as $n \rightarrow+\infty$, such that

$$
\int_{t_{n}-1}^{t_{n}+1} \int_{\Omega}\left|f(s, x)-f_{\infty}(x)\right| \rho(x) \mathrm{d} x \mathrm{~d} s \rightarrow 0 \text { as } n \rightarrow+\infty
$$

and

$$
\int_{t_{n}-1}^{t_{n}+1} \int_{\partial \Omega}\left|h(s, x)-h_{\infty}(x)\right| \mathrm{d} \sigma \mathrm{d} s \rightarrow 0 \text { as } n \rightarrow+\infty .
$$

Assume in addition that

$$
u \in L^{\infty}\left(0, \infty ; L^{1}(\Omega ; \rho)\right) .
$$

Then $u\left(t_{n}, \cdot\right) \rightarrow u_{\infty}$ in $L^{1}(\Omega ; \rho)$ with $u_{\infty}$ the very weak solution of (7) with data $f_{\infty}$ and $h_{\infty}$. 
For the qualitative behaviour of the solutions, we consider now the half plane case under the assumptions

$$
\left\{\begin{array}{l}
\Omega=\mathbb{R} \times[0, \infty), a_{i j} \text { are constants, } f \equiv 0, u_{0} \geq 0, \\
\beta(u) \text { is given by }(8) \text { or }(5), \\
h \in W_{l o c}^{1,1}\left(0,+\infty ; L^{1}(\partial \Omega)\right) \cap L^{\infty}((0,+\infty) \times \partial \Omega), \\
h(t, \cdot)=0 \text { on }(-\infty, 0) \text { and } h(t, \cdot)>0 \text { on }(0,+\infty), \\
h\left(t, x_{1}\right)=c_{+} \forall x_{1} \in[\delta, \infty), t>0 .
\end{array} \quad\left(\widehat{H}_{h p}\right)\right.
$$

In this setting we adapt the definition of limit very weak solution from the elliptic case using truncated-in-space solutions. Existence and uniqueness can be obtain in similar way.

Corollary 1.13. Assume $\left(\widehat{H}_{h p}\right)$. Then there exist four positive constants $\underline{C}<$ $\bar{C}, \underline{\varepsilon} \leq \bar{\varepsilon}$ and two boundary points $\underline{x}_{1, \varepsilon}, \bar{x}_{1, \varepsilon}>0$ such that

i) if $u_{0}=0, h\left(t, x_{1}\right) \leq \underline{C} x_{1}^{\frac{2}{1-q}}$ for a.e. $x_{1} \in\left(0, \underline{x}_{1, \varepsilon}\right)$ and $h\left(t, x_{1}\right) \leq \underline{\varepsilon}$ for a.e. $x_{1} \in\left(\underline{x}_{1, \varepsilon},+\infty\right)$, for any $t \geq 0$, then the non-diffusion on the boundary of the support property holds for any $t \geq 0$, i.e., $\partial \mathcal{S}(u(t, \cdot)) \cap \partial \Omega=\mathcal{S}(h(t, \cdot))$ for any $t \geq 0$ (infinite waiting time property).

ii) Assume that $u_{0}\left(x_{1}, x_{2}\right) \geq u_{1}\left(x_{1}, x_{2}\right)+u_{2}\left(x_{1}, x_{2}\right)$, with $u_{1}$ and $u_{2}$ solutions of the problems (57) and (58), and that $h\left(t, x_{1}\right) \geq \bar{C} x_{1}^{\frac{2}{1-q}}$ for a.e. $x_{1} \in\left(0, \bar{x}_{1, \varepsilon}\right)$ and $h\left(t, x_{1}\right) \geq \bar{\varepsilon}$ for a.e. $x_{1} \in\left(\bar{x}_{1, \varepsilon},+\infty\right)$, for any $t \in(0, T)$.

Then the expansion on the boundary of the support property holds for any $t \in$ $(0, T]$, i.e., $\mathcal{S}(h(t, \cdot)) \subsetneq \partial \mathcal{S}(u(t, \cdot)) \cap \partial \Omega:=\left[-\delta_{0}, \infty\right] \times\{0\}$, for some $\delta_{0}>0$.

Remember that $q \in(0,1)$ when $\beta(u)$ is given by (8) and $q=0$ when $\beta$ is given by 5 .

Corollary 1.14. The conclusions of Corollary 1.6 remain valid for $u(t, \cdot)$ under the corresponding assumptions.

Remark 1.12. For the special case of $q=0$ it is illustrative to compare the conclusions of Theorem 1.5 (respectively Corollary 1.13) with the complementary information given in Theorem B of [30] (respectively Theorem I of $[4])$.

The organization of the rest of the paper is the following. Section 2 is devoted to the proof of the general existence and uniqueness results, Theorems 1.2 and Theorem 1.10. The stabilization of very weak solutions, when $t \rightarrow+\infty$, is considered in Section 3 and, in particular Theorem 1.12 is proved there. Finally the special case of the half plane is considered in Section 4. After proving Theorem 1.4 we present the proof of Theorem 1.5 in Subsections 4.2 and 4.3 . The special case of discontinuous boundary data plays an important role in such proof and so it is previously discussed there. 


\section{On the existence and uniqueness of very weak solutions}

In this part we omit to write the parameter $\lambda$ as it can be thought as already included in $\beta$.

\subsection{Proof of Theorem 1.2}

We need to introduce first a result on the corresponding linear problem

$$
\begin{cases}-L u=f & \text { in } \Omega, \\ u=h & \text { on } \partial \Omega .\end{cases}
$$

Definition 2.1. Assume $f \in L^{1}(\Omega ; \rho)$ and $h \in L^{1}(\partial \Omega)$. A function $u \in L^{1}(\Omega)$ is a weak solution of (23) if it satisfies

$$
\int_{\Omega} u L^{*} \phi \mathrm{d} x=\int_{\partial \Omega} h \partial_{A} \phi \mathrm{d} \sigma-\int_{\Omega} f \phi \mathrm{d} x
$$

for every function $\phi \in C_{0}^{2}(\bar{\Omega})$.

The following lemma is a known result and we cite it for further needs.

Lemma 2.2. Assume that $u$ solves (23) with $h=0$ and $f \in L^{2}(\Omega)$. Then $u \in H_{0}^{1}(\Omega)$ and

$$
\int_{\Omega}-L u \cdot u d x \geq C\|u\|_{L^{2}(\Omega)}^{2}
$$

Next proposition is a consequence of a generalization of the estimates of Brezis [12] applied to the operator $L$. The proof when $L$ is the Laplacian can be found in 26. The case of an even more general second order linear operator of the form

$$
L u=-\operatorname{div}(\mathbf{A} \nabla u)+\mathbf{b} \cdot \nabla u-\operatorname{div}(\mathbf{c} u)+d u,
$$

under appropriate structural and regularity assumptions on the coefficients $\mathbf{A} \in$ $M^{n \times n}(\mathbb{R}), \mathbf{b}, \mathbf{c} \in \mathbb{R}^{n}, d \in \mathbb{R}$ (essentially the maximum principle should hold), is contained in 32 .

Proposition 2.3. Let $f \in L^{1}(\Omega ; \rho)$ and $h \in L^{1}(\partial \Omega)$. Then there exists a unique solution $u \in L^{1}(\Omega)$ of problem (23) in the sense of Definition 2.1. Moreover there exists $C=C(\Omega, L)>0$ such that

$$
\|u\|_{L^{1}(\Omega)} \leq C\left(\|f\|_{L^{1}(\Omega ; \rho)}+\|h\|_{L^{1}(\partial \Omega)}\right)
$$

and $u$ satisfies

$$
-\int_{\Omega} u_{+} L^{*} \phi \mathrm{d} x \leq \int_{\Omega} f(\operatorname{sgn}+u) \phi \mathrm{d} x-\int_{\partial \Omega} \partial_{A} \phi h_{+} \mathrm{d} \sigma
$$

and

$$
-\int_{\Omega}|u| L^{*} \phi \mathrm{d} x \leq \int_{\Omega} f(\operatorname{sgn} u) \phi \mathrm{d} x-\int_{\partial \Omega} \partial_{A} \phi|h| \mathrm{d} \sigma,
$$


for every non negative $\phi \in C_{0}^{2}(\bar{\Omega})$. We have used the notation

$$
\operatorname{sgn} r=\left\{\begin{array}{ll}
1 & \text { if } \mathrm{r}>0, \\
0 & \text { if } \mathrm{r}=0, \\
-1 & \text { if } \mathrm{r}<0,
\end{array} \quad \operatorname{sgn}_{+} r= \begin{cases}1 & \text { if } \mathrm{r} \geq 0, \\
0 & \text { if } \mathrm{r}<0 .\end{cases}\right.
$$

Proof of Theorem 1.2. Uniqueness, monotonicity and estimate $(12)$ follow from Proposition 2.3. Indeed, assume that $u_{1}$ and $u_{2}$ are solutions of (7) with data $f_{1}, h_{1}$ and $f_{2}, h_{2}$ respectively. It means that there exist $b_{1}(x) \in \beta\left(u_{1}(x)\right)$ and $b_{2}(x) \in \beta\left(u_{2}(x)\right)$ such that (11) holds. This implies that $w=u_{1}-u_{2}$ is a solution of

$$
\begin{cases}-L w=f^{*}=f_{1}-f_{2}-b_{1}+b_{2} & \text { in } \Omega, \\ w=h^{*}=h_{1}-h_{2} & \text { on } \partial \Omega .\end{cases}
$$

Then, estimate 12 follows from 25 when applied to $w$ with test function $\phi_{0}$ solution of

$$
\begin{cases}-L^{*} \phi_{0}=1 & \text { in } \Omega, \\ \phi_{0}=0 & \text { on } \partial \Omega .\end{cases}
$$

Also monotonicity follows from estimate (25) when applied to $w$ with test function $\phi_{0}$. Uniqueness can be derived with the same procedure from (26).

Existence. We consider the Yosida approximation $\beta_{\mu}$ of $\beta$, maximal monotone graph of $\mathbb{R}^{2}$, which we know to be a Lipschitz increasing function (see [13]). We look for a solution of the problem

$$
\begin{cases}-L u+\beta_{\mu}(u)=f & \text { in } \Omega, \\ u=h & \text { on } \partial \Omega .\end{cases}
$$

The solution of such a problem is a straightforward generalisation of Proposition 2.1.2 in [26].

Let us call $u_{\mu}$ the solution of $(29)$, and let consider $f \in L^{\infty}(\Omega)$ and $h \in$ $L^{\infty}(\partial \Omega)$. Then, by the monotonicity of solutions, one have the upper bound $u_{\mu} \leq M=\max \left(\sup _{\Omega} f, \sup _{\partial \Omega} h\right)$ for all $\mu>0$. Also $\left\{\beta_{\mu}\left(u_{\mu}\right)\right\}$ is uniformly bounded in $L^{\infty}(\Omega)$ (see [14]).

We now show that $\left\{u_{\mu}\right\}$ and $\left\{\beta_{\mu}\right\}$ are Cauchy sequences in $L^{2}(\Omega)$. Given $\lambda, \mu>0$, we subtract the equations for $u_{\lambda}$ and $u_{\mu}$, multiply the result for $u_{\lambda}-u_{\mu}$ and integrate to obtain, with the use of Lemma 2.2

$$
\begin{aligned}
0 & =\int_{\Omega}-L\left(u_{\lambda}-u_{\mu}\right)\left(u_{\lambda}-u_{\mu}\right)+\int_{\Omega}\left(\beta_{\lambda}\left(u_{\lambda}\right)-\beta_{\mu}\left(u_{\mu}\right)\right)\left(\left(u_{\lambda}-u_{\mu}\right)\right) \\
& \geq C\left\|u_{\lambda}-u_{\mu}\right\|_{L^{2}(\Omega)}^{2}+\left(\beta_{\lambda}\left(u_{\lambda}\right)-\beta_{\mu}\left(u_{\mu}\right),\left(u_{\lambda}-u_{\mu}\right)\right)_{L^{2}(\Omega)},
\end{aligned}
$$

which, following [14], gives

$$
C\left\|u_{\lambda}-u_{\mu}\right\|_{L^{2}(\Omega)}^{2}+\left(\beta_{\lambda}\left(u_{\lambda}\right)-\beta_{\mu}\left(u_{\mu}\right), \lambda \beta_{\lambda}\left(u_{\lambda}\right)-\mu \beta_{\mu}\left(u_{\mu}\right)\right)_{L^{2}(\Omega)} \leq 0 .
$$

Sending $\lambda, \mu \rightarrow 0$ and remembering that $\left\{\beta_{\mu}\left(u_{\mu}\right)\right\}$ is uniformly bounded in $L^{\infty}(\Omega)$, we get that $\left\|u_{\lambda}-u_{\mu}\right\|_{L^{2}(\Omega)} \rightarrow 0$. We set $u:=\lim _{\mu \rightarrow 0} u_{\mu}$. By Lemma 2.4 of [16] also $\beta_{\mu}\left(u_{\mu}\right)$ is a Cauchy sequence in $L^{2}(\Omega)$ and its limit $b \in L^{\infty}(\Omega)$ 
satisfies that $b(x) \in \beta(u(x))$ since $\beta$ is maximal. Passing to the limit in the definition of solution we have

$$
\begin{gathered}
0=-\int_{\Omega} u_{\mu} L^{*} \varphi \mathrm{d} x+\lambda \int_{\Omega} \beta_{\mu}\left(u_{\mu}\right) \varphi \mathrm{d} x-\int_{\Omega} f \varphi \mathrm{d} x+\int_{\partial \Omega} h \partial_{A} \varphi \mathrm{d} \sigma \rightarrow \\
-\int_{\Omega} u L^{*} \varphi \mathrm{d} x+\lambda \int_{\Omega} b \varphi \mathrm{d} x-\int_{\Omega} f \varphi \mathrm{d} x+\int_{\partial \Omega} h \partial_{A} \varphi \mathrm{d} \sigma
\end{gathered}
$$

for any $\varphi \in C_{0}^{2}(\bar{\Omega})$. Hence $u$ is a solution of $(7)$.

If $(f, h) \in L^{1}(\Omega ; \rho) \times L^{1}(\partial \Omega)$ we consider $\left\{\left(f_{n}, h_{n}\right)\right\} \subset L^{\infty}(\Omega) \times L^{\infty}(\partial \Omega)$ which converges to $(f, h)$ in $L^{1}(\Omega ; \rho) \times L^{1}(\partial \Omega)$. Call $u_{n}$ the solution of $(7)$ with data $f_{n}$ and $h_{n}$. Thanks to (13), $u_{n}$ and $b_{n}$ are Cauchy sequences in $L^{1}(\Omega)$ and hence converges to functions $u, b$ respectively. Since $\beta$ is maximal $b(x) \in \beta(u(x))$ and passing to the limit in the definition of solution we find that $u$ solves (7).

\subsection{Proof of Theorem 1.10}

We start this part by giving a result on the corresponding linear problem

$$
\begin{cases}u_{t}-L u=f(t, x) & \text { in } Q_{T}, \\ u=h(t, x) & \text { on } \Sigma_{T}, \\ u(0, x)=u_{0}(x) & \text { on } \Omega .\end{cases}
$$

Proposition 2.4. Assume $f \in L^{1}\left(Q_{T} ; \rho\right)=L^{1}\left(0, T ; L^{1}(\Omega ; \rho)\right), h \in L^{1}\left(\Sigma_{T}\right)$ and $u_{0} \in L^{1}(\Omega ; \rho)$. Problem (31) possesses a unique very weak solution $u \in L^{1}\left(Q_{T}\right)$, in the sense that

$$
\int_{Q_{T}}-\left(\zeta_{t}+L \zeta\right) u-f \zeta \mathrm{d} x \mathrm{~d} t=-\int_{\Sigma_{T}} h \partial_{A} \zeta \mathrm{d} \sigma \mathrm{d} t+\int_{\Omega} \zeta(x, 0) u_{0} \mathrm{~d} x
$$

for every $\zeta \in C^{2,1}\left(\bar{Q}_{T}\right)$. Additionally, there hold

$$
\begin{aligned}
\int_{Q_{T}}-\left(\zeta_{t}+L \zeta\right) u_{+}-f \zeta \operatorname{sgn}_{+}(u) \mathrm{d} x \mathrm{~d} t & \\
& \leq-\int_{\Sigma_{T}} h_{+} \partial_{A} \zeta \mathrm{d} \sigma \mathrm{d} t+\int_{\Omega} \zeta(x, 0)\left(u_{0}\right)_{+} \mathrm{d} x
\end{aligned}
$$

and

$$
\begin{aligned}
& \int_{Q_{T}}-\left(\zeta_{t}+L \zeta\right)|u|-f \zeta \operatorname{sgn}(u) \mathrm{d} x \mathrm{~d} t \\
& \leq-\int_{\Sigma_{T}}|h| \partial_{A} \zeta \mathrm{d} \sigma \mathrm{d} t+\int_{\Omega} \zeta(x, 0)\left|u_{0}\right| \mathrm{d} x
\end{aligned}
$$

for every non-negative $\zeta \in C_{0}^{2,1}\left(Q_{T}\right)$.

Proof. The proof is exactly the same of [25] with $\Delta$ replaced by $L$.

Lemma 2.5. Let $f \in L^{1}\left(Q_{T} ; \rho\right), h \in L^{1}\left(\Sigma_{T}\right)$ and $u_{0} \in L^{1}(\Omega ; \rho)$. Then problem (9) has at most one solution. If $u_{1}, u_{2}$ are solutions with data $f_{1}, h_{1}, u_{01}$ and $f_{2}, h_{2}, u_{02}$ respectively, then

$$
\begin{aligned}
& \left\|u_{1}-u_{2}\right\|_{L^{1}\left(Q_{T}\right)}+\left\|b_{1}-b_{2}\right\|_{L^{1}\left(Q_{T} ; \rho\right)} \\
& \leq C\left(\left\|f_{1}-f_{2}\right\|_{L^{1}\left(Q_{T} ; \rho\right)}+\left\|h_{1}-h_{2}\right\|_{L^{1}\left(\Sigma_{T}\right)}+\left\|u_{01}-u_{02}\right\|_{L^{1}(\Omega ; \rho)}\right),
\end{aligned}
$$


with $C>0$ and $b_{1} \in \beta\left(u_{1}\right), b_{2} \in \beta\left(u_{2}\right)$. Furthermore, if $f_{1} \leq f_{2}, h_{1} \leq h_{2}$ and $u_{01} \leq u_{02}$ then $u_{1} \leq u_{2}$.

Proof. As for the elliptic case, we set $w=u_{1}-u_{2}$, which is solution of

$$
\begin{cases}w_{t}-L w=f_{1}-f_{2}-b_{1}+b_{2} & \text { in } \mathrm{Q}_{\mathrm{T}}, \\ w=h_{1}-h_{2} & \text { on } \Sigma_{\mathrm{T}}, \\ w(0, x)=u_{01}-u_{02} & \text { in } \Omega .\end{cases}
$$

Take $\psi_{T}$ solution of

$$
\begin{cases}-\psi_{t}-L \psi=1 & \text { in } \mathrm{Q}_{\mathrm{T}}, \\ \psi=0 & \text { on } \Sigma_{\mathrm{T}}, \\ \psi(T, x)=0 & \text { in } \Omega .\end{cases}
$$

Estimate (34) and monotonicity follow from 32 with $\psi_{T}$ as test function and uniqueness follows from (33) with the same method.

Proof of Theorem 1.10. The proof of existence for the case of $\beta$ a continuous function is again an easy adaptation of previous results in the literature concerning the special case of $L=\Delta$ (see, e.g., 24] Lemma 2.7 and [25] Lemma 1.3 and Lemma 1.7: notice that the assumption of $\beta$ Lipschitz assumed at the beginning of the paper is not needed in both lemmas). The adaptation to the case of $L$ given by (6) and $\beta$ multivalued is completely similar to the one presented in the stationary case.

The continuity in $t$ of the very weak solution will be used in our study of the asymptotic behaviour of solutions and can be obtained by reformulating the parabolic semilinear problem as an abstract Cauchy problem on the Banach space $X=L^{1}(\Omega ; \rho)$,

$$
(A P)\left\{\begin{array}{l}
\frac{d u}{d t}(t)+A(t) u(t) \ni f(t) \quad \text { in } X, \\
u(0)=u_{0},
\end{array}\right.
$$

where $A(t): D(A(t)) \rightarrow \mathcal{P}(X)$ is the operator defined by $(w, z) \in A(t) \subset X \times X$ iff $w \in L^{1}(\Omega)$ is the very weak solution of

$$
\begin{cases}-L w+\beta(w) \ni z & \text { in } \Omega, \\ w(x)=h(t, x) & \text { on } \partial \Omega .\end{cases}
$$

Here, in the definition of the operator $A(t), t \in(0, T)$ is a parameter (remember that $h \in W^{1,1}\left(0, T ; L^{1}(\partial \Omega)\right)$, hence $h(t, \cdot)$ makes sense). For a.e. $t \in(0, T)$, this operator is $T-\omega$-accretive on $X$ (see, e.g., 8 or 10 ) for some $\omega \geq 0$ large enough. Indeed, we must show that, for some $\mu>0,(I+\mu(A(t)+\omega I))^{-1}$ is a contraction on $X$ and this is equivalent to show that if $w^{i}$, with $i=1,2$, are the very weak solutions of

$$
\begin{cases}-\mu L w^{i}+\mu \beta\left(w^{i}\right)+\mu \omega w^{i} \ni z^{i}(x) & \text { in } \Omega, \\ w^{i}(x)=h(t, x) & \text { on } \partial \Omega,\end{cases}
$$

for some $z^{i} \in L^{1}(\Omega ; \rho)$, then

$$
\left\|\left[w^{1}-w^{2}\right]_{+}\right\|_{L^{1}(\Omega ; \rho)} \leq\left\|\left[z^{1}-z^{2}\right]_{+}\right\|_{L^{1}(\Omega ; \rho)} .
$$


But this is a trivial consequence of the estimates proven in Theorem 1.2 once that $\omega$ is taken large enough (in particular $\omega>C$, the constant appearing in estimate 12 which was only dependent on $\Omega$ ). In addition, for a.e. $t \in$ $(0, T)$ this operator is m-accretive (see [8]) in the sense that $R(I+\mu A(t))=X$. Indeed, we must prove that problem (35) (i.e., (7)) for a given right hand side in $z^{i} \in L^{1}(\Omega ; \rho)$ has a unique solution, which, again, is a consequence of Theorem 1.2. Finally, since $h \in W^{1,1}\left(0, T ; L^{1}(\partial \Omega)\right)$ we get that the $t$-dependence of the solution has the same regularity: $w \in W^{1,1}\left(0, T ; L^{1}(\Omega)\right)$ and so the CrandallEvans theorem $([15],[21])$ can be applied ensuring the existence and uniqueness of a mild solution $u \in C\left([0, T] ; L^{1}(\Omega ; \rho)\right)$ of the abstract problem $(A P)$. Finally, since we have uniqueness of the very weak solution of the parabolic problem, it is easy to see (as, for instance, in [11]) that both solutions must coincide and thus we get the desired time regularity result.

\section{On the stabilization when $t \rightarrow+\infty$}

Remember that the solutions of the parabolic problem 9 and the elliptic problem (7) will be indicated with $u$ and $u_{\infty}$ respectively.

Proof of Theorem 1.12. We follow some of the ideas contained in 18, (see also references therein). We define

$$
U_{n}(s, x)=u\left(t_{n}+s, x\right), \quad F_{n}(s, x)=f\left(t_{n}+s, x\right), \quad H_{n}(s, x)=h\left(t_{n}+s, x\right),
$$

where $t_{n} \rightarrow \infty$ when $n \rightarrow \infty$. By Theorem 1.10 we know that there exists $b \in L^{1}\left(0, T ; L^{1}(\Omega ; \rho)\right)$ such that $b(t, x) \in \beta(u(t, x))$ a.e. in $Q_{T}$. Thus we also define $B_{n}(s, x)=b\left(t_{n}+s, x\right)$. Then it is clear that

$$
\begin{cases}\frac{\partial U_{n}}{\partial s}-L U_{n}+\lambda B_{n}(s, x)=F_{n}(s, x) & \text { in }(-1,1) \times \Omega \\ U_{n}=H_{n}(s, x) & \text { on }(-1,1) \times \partial \Omega .\end{cases}
$$

for all $n>1$. Then from the estimate of Theorem 1.10 (which coincides with (1.26) of [25] easily adapted to the case in which $L$ is given by (6)),

$$
\begin{aligned}
& \left\|U_{n}\right\|_{L^{1}((-1,1) \times \Omega)}+\lambda\left\|B_{n}\right\|_{L^{1}\left((-1,1) ; L^{1}(\Omega ; \rho)\right)} \\
& \quad \leq C\left(\left\|F_{n}\right\|_{L^{1}\left((-1,1) ; L^{1}(\Omega ; \rho)\right)}+\left\|H_{n}\right\|_{L^{1}\left((-1,1) ; L^{1}(\partial \Omega)\right)}+\left\|U_{n}(-1, \cdot)\right\|_{\left.L^{1}(\Omega ; \rho)\right)}\right) .
\end{aligned}
$$

Assumptions (20) and (21) imply that $F_{n} \rightarrow F_{\infty}$, and $H_{n} \rightarrow H_{\infty}$ strongly in $L^{1}\left((-1,1) ; L^{1}(\Omega ; \rho)\right)$ and $L^{1}\left((-1,1) ; L^{1}(\partial \Omega)\right)$ respectively, where $F_{\infty}$ and $H_{\infty}$ are defined as $F_{\infty}(s, x)=f_{\infty}(x)$ and $H_{\infty}(s, x)=H_{\infty}(x)$. Moreover, from assumption (22), $\left\|U_{n}(-1, \cdot)\right\|_{\left.L^{1}(\Omega ; \rho)\right)}$ is bounded independently of $n$. Consequently, due to assumption $(19),\left\{\rho B_{n}\right\}$ is a bounded sequence in $L^{p}\left((-1,1) ; L^{p}(\Omega)\right)$ with $p=1 / q$ if $q \in(0,1)$ and for every $p>1$ if $q=0$. Thus,

$$
\rho B_{n} \rightarrow \rho B_{\infty}
$$

weakly in $L^{p}\left((-1,1) ; L^{p}(\Omega)\right)$ (after passing to a subsequence), for some $B_{\infty}$ in $L^{p}\left((-1,1) ; L^{p}(\Omega)\right)$. Then, by $[25$ (Lemma 1.6 (ii) easily adapted to the case in which $L \neq \Delta)$, we get that $U_{n} \rightarrow U_{\infty}$ (strongly) in $C\left([-1+\varepsilon, 1] ; L^{1}(\Omega ; \rho)\right.$ ) 
for some $U_{\infty} \in C\left([-1+\varepsilon, 1] ; L^{1}(\Omega ; \rho)\right)$ for any $\varepsilon \in(0,2)$. Indeed, since problem (36) is linear we can use the decomposition

$$
U_{n}=\mathbb{P}\left(F_{n}-\lambda B_{n}, 0,0\right)+\mathbb{P}\left(0, H_{n}, 0\right)+\mathbb{P}\left(0,0, U_{n}(-1)\right),
$$

where $\mathbb{P}$ is the solution mapping (see 25] Lemma 1.6). The operator $\mathbb{P}\left(F_{n}-\right.$ $\left.\lambda B_{n}, 0,0\right)$ is compact from $L^{p}((-1+\varepsilon, 1) \times \Omega) \times\{0\} \times\{0\} \rightarrow C([-1+\varepsilon, 1] \times \Omega)$, since in general $w=\mathbb{P}(q, 0,0)$ is given by

$$
w(s, x)=\int_{-1}^{1} \int_{\Omega} G_{L}(x, y, s, \tau) q(y, \tau) \mathrm{d} y \mathrm{~d} \tau
$$

(remember that the Green function $G_{L}(x, \cdot, s, \cdot) \in C_{0}([-1+\varepsilon, 1] \times \bar{\Omega})$. The compactness of the other terms $\mathbb{P}\left(0, H_{n}, 0\right)$ and $\mathbb{P}\left(0,0, U_{n}(-1)\right)$ was shown in Lemma 1.6 (ii) of the mentioned reference. In our case, we know the continuity in time of the functions (Proposition 1.10). In particular, since $U_{n} \rightarrow U_{\infty}$ in $C\left([-1+\varepsilon, 1] ; L^{1}(\Omega)\right)$, we find that $\left\{U_{n}(-1+\varepsilon, \cdot)\right\}$ is a Cauchy sequence in $L^{1}(\Omega ; \rho)$.

Then, by applying an estimate similar to 13 but for the parabolic problem (see estimate (1.26) of 25 easily adapted to the case in which $L$ is given by (6)), we get

$$
\begin{aligned}
& \left\|U_{n}-U_{m}\right\|_{L^{1}((-1+\varepsilon, 1) \times \Omega)}+\lambda\left\|B_{n}-B_{m}\right\|_{L^{1}\left((-1+\varepsilon, 1) ; L^{1}(\Omega ; \rho)\right)} \\
& \leq C\left(\left\|F_{n}-F_{m}\right\|_{L^{1}\left((-1+\varepsilon, 1) ; L^{1}(\Omega ; \rho)\right)}+\left\|H_{n}-H_{m}\right\|_{L^{1}\left((-1+\varepsilon, 1) ; L^{1}(\partial \Omega)\right)}\right. \\
& \left.\quad+\left\|U_{n}(-1+\varepsilon)-U_{m}(-1+\varepsilon)\right\|_{L^{1}(\Omega ; \rho)}\right)
\end{aligned}
$$

which proves that $\left\{\rho B_{n}\right\}$ is a Cauchy sequence in $L^{1}\left((-1+\varepsilon, 1) ; L^{1}(\Omega)\right)$ and so $\rho B_{n} \rightarrow \rho B_{\infty}$ strongly in $L^{1}\left((-1+\varepsilon, 1) ; L^{1}(\Omega)\right)$. Then, since $\beta$ is maximal monotone, we conclude (see [9]) that $B_{\infty}(s, x) \in \beta\left(U_{\infty}(s, x)\right)$ for a.e. $(s, x) \in$ $(-1+\varepsilon, 1) \times \Omega$.

It only remains to prove that $U_{\infty}(s, x)=u_{\infty}(x)$, with $u_{\infty}$ the (unique) very weak solution of (7) with data $f_{\infty}, h_{\infty}$. Since

$$
\text { ess } \sup _{t_{n}+s \in(0,+\infty)}\left\|u\left(t_{n}+s, .\right)\right\|_{L^{1}(\Omega ; \rho)} \leq C
$$

then there exists a (stationary) Radon measure $\mu_{\infty} \in M(\Omega ; \rho)$ such that $u\left(t_{n}+\right.$ $s, \cdot) \rightarrow \mu_{\infty}$ weakly in $M(\Omega ; \rho)$. Moreover, $u\left(t_{n}+s, \cdot\right) \rightarrow U_{\infty}(s, x)$ (strongly) in $C\left([-1+\varepsilon, 1] ; L^{1}(\Omega ; \rho)\right) \subset C([-1+\varepsilon, 1] ; M(\Omega ; \rho))$. Then, by the uniqueness of the limit, we deduce that $U_{\infty}(s, \cdot)=\mu_{\infty}(\cdot)$ for any $s \in[-1+\varepsilon, 1]$, so that the singular part of the measure $\mu_{\infty}(\cdot)$ vanishes (i.e., $\left.\mu_{\infty} \in L^{1}(\Omega ; \rho)\right)$. Let us denote now $u_{\infty}(x) \equiv \mu_{\infty}(x)$. Then $U_{\infty}(s, x)=u_{\infty}(x)$ for any $s \in[-1+\varepsilon, 1]$. By the same reasons (thanks to the assumption on $\beta$ ) we get that $B_{\infty}(s, x)=b_{\infty}(x)$ for a.e. $s \in[-1+\varepsilon, 1]$, for some $b_{\infty} \in L^{1}(\Omega ; \rho)$ such that $b_{\infty}(x) \in \beta\left(u_{\infty}(x)\right)$ for a.e. $x \in \Omega$. Finally, we take as test function in the definition of very weak solution of the parabolic problem $\varphi(s, x)=\psi(s) \zeta(x)$, with $\zeta \in W^{2, \infty}(\Omega) \cap$ $W_{0}^{1, \infty}(\Omega)$ and $\psi \in C^{1}([-1,1])$ such that $\psi_{\mid[-1,-1+\varepsilon]}=\psi(1)=0$ and such that $\int_{-1+\varepsilon}^{+1} \psi(s) d s=1$. Obviously such special $\varphi(s, x)$ is a correct test function since $\varphi \in W^{1, \infty}\left(-1,1 ; L^{\infty}(\Omega)\right) \cap L^{\infty}\left(-1,1 ; W^{2, \infty}(\Omega) \cap W_{0}^{1, \infty}(\Omega)\right)$ and $\varphi(1, \cdot)=0$.

Then, from the definition of very weak solution, we get that 


$$
\begin{gathered}
-\int_{-1+\varepsilon}^{1} \int_{\Omega} U_{n}(s, x) \psi^{\prime}(s) \zeta(x) \mathrm{d} x \mathrm{~d} s+\int_{-1+\varepsilon}^{1} \int_{\Omega} \psi(s) U_{n}(s, x) L^{*} \zeta(x) \mathrm{d} x \mathrm{~d} s \\
+\lambda \int_{-1+\varepsilon}^{1} \int_{\Omega} B_{n}(s, x) \psi(s) \zeta(x) \mathrm{d} x \mathrm{~d} s \\
=\int_{\Omega} U_{n}(-1, x) \psi(-1) \zeta(x) \mathrm{d} x+\int_{-1+\varepsilon}^{1} \int_{\Omega} F_{n}(s, x) \psi(s) \zeta(x) \mathrm{d} x \mathrm{~d} s \\
-\int_{-1+\varepsilon}^{1} \int_{\partial \Omega} \psi(s) H_{n}(s, \sigma) \frac{\partial \zeta(\sigma)}{\partial n} \mathrm{~d} \sigma \mathrm{d} s .
\end{gathered}
$$

Passing to the limit, as $n \rightarrow+\infty$, and using that $\psi(-1+\varepsilon)=0$, we arrive to

$$
\begin{aligned}
& -\left(\int_{-1+\varepsilon}^{1} \psi^{\prime}(s) \mathrm{d} s\right)\left(\int_{\Omega} u_{\infty}(x) \zeta(x) \mathrm{d} x\right)+\int_{-1+\varepsilon}^{1} \psi(s) \mathrm{d} s \int_{\Omega} u_{\infty}(x) L^{*} \zeta(x) \mathrm{d} x \\
& \quad+\lambda \int_{-1+\varepsilon}^{1} \int_{\Omega} B_{\infty}(s, x) \psi(s) \zeta(x) \mathrm{d} x \\
& =\int_{-1+\varepsilon}^{1} \psi(s) \mathrm{d} s \int_{\Omega} f_{\infty}(x) \zeta(x) \mathrm{d} x-\int_{-1+\varepsilon}^{1} \psi(s) \mathrm{d} s \int_{\partial \Omega} h_{\infty}(\sigma) \frac{\partial \zeta(\sigma)}{\partial n} \mathrm{~d} \sigma .
\end{aligned}
$$

But

$$
\int_{-1+\varepsilon}^{1} \psi^{\prime}(s) \mathrm{d} s=0,
$$

and since $\int_{-1+\varepsilon}^{+1} \psi(s) \mathrm{d} s=1$ we get that

$$
\begin{aligned}
& \int_{\Omega} u_{\infty}(x) L^{*} \zeta(x) \mathrm{d} x+\lambda \int_{\Omega} b_{\infty}(x) \zeta(x) \mathrm{d} x \mathrm{~d} s \\
&=\int_{\Omega} f_{\infty}(x) \psi(s) \zeta(x) \mathrm{d} x-\int_{\partial \Omega} h_{\infty}(\sigma) \frac{\partial \zeta(\sigma)}{\partial n} \mathrm{~d} \sigma
\end{aligned}
$$

which shows that $u_{\infty}$ coincides with the (unique) very weak solution of the stationary problem.

Remark 3.1. Notice that the boundedness of the trajectories assumption is considerably weaker than the usual for weak solutions (see, e.g, [18] which is of the type $u \in L^{\infty}\left(0,+\infty ; H^{1}(\Omega)\right)$. Notice also that this condition is necessary once we assume that the conclusion of Theorem 1.12 holds.

A sufficient condition leading to the boundedness of the trajectories (assumption (22) can be obtained by the method of super and subsolutions as in Proposition 3 of [18]. 
Proposition 3.2. Assume that the stationary problem (7) admits a bounded weak solution $u_{\infty}$. Let $f, f_{\infty}$ and $h, h_{\infty}$ satisfy (20) and (21) respectively. Suppose the existence of $f_{u}, f_{d} \in L^{1}((0, T) \times \Omega)$ for any $T>0$ and $h_{u}, h_{d} \in$ $L^{1}((0, T) \times \partial \Omega)$ with $f_{u}, h_{u}\left(f_{d}, h_{d}\right)$ non-increasing (decreasing) in $t$ such that

$$
\begin{aligned}
& -\bar{f}(x) \leq f_{d}(t, x) \leq f(t, x) \leq f_{u}(t, x) \leq \bar{f}(x), \\
& -\bar{h}(x) \leq h_{d}(t, x) \leq h(t, x) \leq h_{u}(t, x) \leq \bar{h}(x),
\end{aligned}
$$

for $0 \leq \bar{f}=\operatorname{div} \mathbf{c}$ with $\mathbf{c} \in L^{p}(\Omega)^{N}, 0 \leq \bar{h} \in L^{1}(\partial \Omega)$ and

$$
\begin{aligned}
& \lim _{t \rightarrow \infty} f_{u}(t, x)=\lim _{t \rightarrow \infty} f_{d}(t, x)=f_{\infty}(x) \text { in } L^{1}(\Omega ; \rho), \\
& \lim _{t \rightarrow \infty} h_{u}(t, x)=\lim _{t \rightarrow \infty} h_{d}(t, x)=h_{\infty}(x) \text { in } L^{1}(\partial \Omega) .
\end{aligned}
$$

Let $u, u_{u}, u_{d}$ be bounded weak solutions of (9) associated to the data $\left(f, h, u_{0}\right)$, $\left(f_{u}, h_{u}, \bar{u}_{0}\right)$ and $\left(f_{d}, h_{d}, \underline{u}_{0}\right)$ with $\bar{u}_{0}, \underline{u}_{0}$ solutions of (7) with data $\bar{f}, \bar{h}$ and $-\bar{f},-\bar{h}$ respectively. If $u_{u}, u_{d} \in L^{\infty}\left(\left(t_{0}, \infty\right) ; L^{1}(\Omega)\right)$ for some $t_{0}>0$ then the conclusion of Theorem 1.12 holds.

\section{On the half plane problems}

Before starting with proving existence and uniqueness we shall show some results concerning the boundedness of the support of solutions. For this purpose we assume that

$$
\beta(u)=u^{q}, \quad \lambda=1, \quad \sup h=1 .
$$

This hypothesis on $\lambda$ and $h$ is not a restriction as, taking it off, all calculations can be performed in the same way. Moreover the proof of Theorems 1.4 and 1.5 for the multivalued case (which formally corresponds to make $q=0$ in all the above expressions) follows, word by word, the same proof of the case in which $q \in(0,1)$ and replacing the identity symbol $=$ by the one of containing $\ni$. The details about local super and subsolutions can be seen also in the book [17] (Theorem 2.16, Chapter 2).

In this first part we study some comparison functions which are essential, first of all, to give sense to the formulation of problem 15 , especially to its boundary conditions, fundamental in the definition of "limit very weak solution". Secondly, they are important also in the study of the behaviour of solutions near the origin.

Assuming (38), we repeat the same procedure of [20], looking for local supersolutions, which are solutions of

$$
\begin{cases}-L u+u^{q}=0 & \text { in } B_{R}\left(x_{0}\right), \\ u=1 & \text { on } \partial B_{R}\left(x_{0}\right),\end{cases}
$$

where $B_{R}\left(x_{0}\right)$ is the ball with radius $R$ and centered in $x_{0}$. The problem is that, differently from [20, we do not know exact radial solution for (39). So we introduce a family of radial supersolution for (39).

Assume that $y(x)=\eta\left(\left|x-x_{0}\right|\right)$ is a radially symmetric function defined in $B_{R}\left(x_{0}\right)$. Then, if we denote with $r=\left|x-x_{0}\right|$, we have 


$$
L y(x)=\eta^{\prime \prime} \sum_{i j} a_{i j} \frac{x_{i} x_{j}}{r^{2}}+\frac{\eta^{\prime}}{r}\left(\sum_{i} a_{i i}-\sum_{i j} a_{i j} \frac{x_{i} x_{j}}{r^{2}}\right) .
$$

Considering that

$$
\sum_{i} a_{i i}-\nu \leq \sum_{i} a_{i i}-\sum_{i j} a_{i j} \frac{x_{i} x_{j}}{r^{2}} \leq \sum_{i} a_{i i}-\mu,
$$

we can define the quantity

$$
B_{A}=\sup _{x \in \Omega}\left(\sum_{i} a_{i i}-\sum_{i j} a_{i j} \frac{x_{i} x_{j}}{r^{2}}\right) .
$$

In particular, if we assume $\eta^{\prime}, \eta^{\prime \prime} \geq 0$,

$$
-L y \geq-\nu \eta^{\prime \prime}-\frac{B_{A}}{r} \eta^{\prime} .
$$

We introduce the operator

$$
L_{\nu}(\eta)=\nu \eta^{\prime \prime}+\frac{B_{A}}{r} \eta^{\prime},
$$

which operates on functions of a real scalar variable and we study the properties of the solutions to the problem

$$
\left\{\begin{array}{l}
-L_{\nu} \eta+\eta^{q}=0 \quad r \in(0, R) \\
\eta(0)=0, \eta(R)=1,
\end{array}\right.
$$

whenever $R \in \mathbb{R}^{+}$. We set the constants

$$
C_{0}=\left(\frac{(1-q)^{2}}{2 \nu(q+1)+2 B_{A}(1-q)}\right)^{1 /(1-q)}, \quad R_{0}=\frac{1}{C_{0}^{(1-q) / 2}}
$$

and introduce the function

$$
\eta_{0}(r)=C_{0} r^{\frac{2}{1-q}}, \quad r \in\left[0, R_{0}\right] .
$$

It is a direct computation to see that $\eta_{0}$ is the solution of 44. For $R>R_{0}$ we do not know the analytic form of the solution $\eta_{R}$ of (44) but we know that the function

$$
\bar{\eta}_{R}(r)= \begin{cases}0 & r \in\left[0, R-R_{0}\right], \\ \eta_{0}\left(r-\left(R-R_{0}\right)\right) & r \in\left[R-R_{0}, R\right],\end{cases}
$$

is a supersolution. The next lemma gives the proof of this fact.

Lemma 4.1. The function $\bar{\eta}_{R}$, for $R>R_{0}$, defined by (46) is a supersolution of (44). 
Proof. For $r \in\left(0, R-R_{0}\right),-L_{\nu} \bar{\eta}_{R}+\bar{\eta}_{R}^{q}=0$. For $r \in\left(R-R_{0}, R\right)$, calling $s=r-\left(R-R_{0}\right)$,

$$
\begin{aligned}
-L_{\nu} \bar{\eta}_{R}+\bar{\eta}_{R}^{q} & =-\nu \eta_{0}^{\prime \prime}(s)-\frac{B_{A}}{s+R-R_{0}} \eta_{0}^{\prime}(s)+\eta_{0}^{q}(s) \\
& \geq-\nu \eta_{0}^{\prime \prime}(s)-\frac{B_{A}}{s} \eta_{0}^{\prime}(s)+\eta_{0}^{q}(s) \\
& =-L_{\nu} \eta_{0}+\eta_{0}^{q}=0,
\end{aligned}
$$

where the inequality is due to the fact that $\eta_{0}^{\prime} \geq 0$ and $R-R_{0} \geq 0$. This inequality combined with the values of $\bar{\eta}_{R}$ at the boundary, i.e., $\bar{\eta}_{R}(0)=0$ and $\bar{\eta}_{R}(R)=1$, makes of $\bar{\eta}_{R}$ a supersolution for problem 44.

The following lemma is the conclusion of this line of reasoning.

Lemma 4.2. Suppose that $u_{R}$ is solution of $(39)$. Then the function $y_{R}(x)=$ $\bar{\eta}_{R}\left(\left|x-x_{0}\right|\right)$ satisfies $y_{R} \geq u_{R}$ in $\bar{B}_{R}\left(x_{0}\right)$.

Proof. The proof uses the comparison principle. Just notice, recalling (42) and Lemma 4.1, that

$$
-L y_{R}+y_{R}^{q} \geq-L_{\nu} \bar{\eta}_{R}+\bar{\eta}_{R}^{q} \geq 0=-L u_{R}+u_{R}^{q}
$$

Checking the boundary conditions we obtain the statement.

Using the results on local supersolutions just given, we can find a function $\rho:\left(R_{0}, \infty\right) \rightarrow \mathbb{R}^{+}$such that if $R>R_{0}$ and if $\eta_{R}$ is the solution of 44 in $(0, R)$ then

$$
\eta_{R}=0 \text { in }[0, \rho(R)] \text { and } \eta_{R}>0 \text { in }(\rho(R), R] .
$$

We define the function

$$
d(R)=R-\rho(R), \quad R>R_{0},
$$

and the following properties hold:

Lemma 4.3. It holds

$$
\begin{aligned}
& \text { (i) } R_{0}<R_{1}<R_{2} \Rightarrow d\left(R_{1}\right) \geq d\left(R_{2}\right) \\
& \text { (ii) } \lim _{R \rightarrow \infty} d(R)=c_{\nu q}
\end{aligned}
$$

with

$$
c_{\nu q}=\frac{(2 \nu(1+q))^{1 / 2}}{1-q} .
$$

Proof. Point $(i)$ is exactly the same as in 20. The second statement follows the same line too but we sketch it to show the details. We already know that on $[0, \rho(R)]$ the solution is zero since $\bar{\eta}_{R}$ is zero. We focus on the problem

$$
\left\{\begin{array}{l}
-L_{\nu} \eta+\eta^{q}=0 \quad r \in(\rho(R), R), \\
\eta(\rho(R))=0, \quad \eta(R)=1 .
\end{array}\right.
$$


We set $w(r)=\eta(R-r)$ and we transform the problem into

$$
\left\{\begin{array}{l}
-\nu w^{\prime \prime}+\frac{B_{s}}{R-r} w^{\prime}+w^{q}=0 \quad \text { in }\left(0, d\left(R_{0}\right)\right), \\
w \geq 0, \\
w(0)=1, \quad w\left(d\left(R_{0}\right)\right)=0 .
\end{array}\right.
$$

We note that the second extreme of the domain in 490 should be $d(R)=$ $R-\rho(R)$, which, according to point $(i)$, is smaller than $d\left(R_{0}\right)$. This does not affect the result since $w=0$ in $\left(d(R), d\left(R_{0}\right)\right)$. We multiply (49) by $w^{\prime}$ and integrate over $\left(r, d\left(R_{0}\right)\right)$

$$
\begin{aligned}
0 & =-\frac{\nu}{2} \int_{r}^{d\left(R_{0}\right)} \frac{d}{d s}\left(w^{\prime}\right)^{2} \mathrm{~d} s+\int_{r}^{d\left(R_{0}\right)} \frac{B_{s}}{R-r}\left(w^{\prime}\right)^{2} \mathrm{~d} s+\frac{1}{q+1} \int_{r}^{d\left(R_{0}\right)} \frac{d}{d s} w^{q+1} \mathrm{~d} s \\
& \geq \frac{\nu}{2}\left(w^{\prime}\right)^{2}(r)-\frac{1}{q+1} w^{q+1}(r) .
\end{aligned}
$$

The inequality in the second line is due to the non-negativity of the second term of the right-hand side in the first line. Since $0 \leq w \leq 1$ we conclude that

$$
\left|w^{\prime}(r)\right| \leq\left(\frac{2}{\nu(q+1)}\right)^{1 / 2}, \quad 0 \leq r \leq d\left(R_{0}\right) .
$$

We have found that the family of solutions $\left\{w(r, R): R>R_{0}\right\}$ is equicontinuous in $\left[0, d\left(R_{0}\right)\right]$ and from compactness argument we can extract a subsequence $\left\{w\left(\cdot, R_{n}\right)\right\}$ with $R_{n} \rightarrow \infty$ and a function $\bar{w} \in C([0, \infty))$ such that $w\left(\cdot, R_{n}\right) \rightarrow \bar{w}$ uniformly on compact sets. Actually what really matters is the convergence on $\left[0, d\left(R_{0}\right)\right]$ as $w(r, R)=0$ for $r>d\left(R_{0}\right)$ and $R>R_{0}$. In the limit, the equation for $\bar{w}$ becomes

$$
\left\{\begin{array}{l}
-\nu w^{\prime \prime}+w^{q}=0 \\
w \geq 0 \\
w(0)=1, \quad w(\infty)=0,
\end{array}\right.
$$

whose unique solution is

$$
z(t)=\left[\left(1-\frac{t}{c_{\nu q}}\right)_{+}\right]^{2 /(1-q)} .
$$

The convergence of $w(\cdot, R)$ to the solution of 50 implies that $d(R) \rightarrow c_{\nu q}$ as $R \rightarrow \infty$.

Lemma 4.4. Let $u_{R}$ be the solution of (39). Then $u_{R}(x) \leq y_{R}(x)=\eta_{R}(\mid x-$ $\left.x_{0} \mid\right)$.

Remark 4.5. If in (39) we set the value at the boundary to be $\varepsilon$ instead of one, all results just shown change just in the value of the constants. To be more specific, the constant $C_{0}$ appearing in (45) remains the same while $R_{0}$ should be changed into $R_{\varepsilon}=\varepsilon^{(1-q) / 2} C_{0}$ and $c_{\nu q}$ in $c_{\nu q \varepsilon}=\varepsilon^{(1-q) / 2} c_{\nu q}$.

We remind that the Heaviside function $H_{v}$ is given by

$$
H_{v}\left(x_{1}\right)= \begin{cases}0 & x_{1} \in(-\infty, 0), \\ 1 & x_{1} \in(0, \infty) .\end{cases}
$$


Lemma 4.6. Let $u$ be a solution of (7) in the setting of $H_{h p}$ with $h$ the Heaviside function. Then

$$
\mathcal{S}(u) \subset\left\{(x, y) \in \Omega: x \geq 0, y<c_{\nu q}\right\} \cup\left\{(x, y) \in \Omega: x \leq 0, r<c_{\nu q}\right\} .
$$

Proof. The proof is the same as the one proposed in [20] and uses the technique of local supersolutions. We start by giving a bound in the $x_{2}$ direction. For $R>R_{0}$ we consider the function $y_{R}$ as in Lemma 4.4 and we set

$$
\bar{u}\left(x_{1}, x_{2} ; \xi\right)=y_{R}\left(x_{1}-\xi, x_{2}-R\right)
$$

defined in $B_{R}(\xi, R)$. Since $\bar{u}=1$ in $\partial B_{R}(\xi, R)$, by the comparison principle we obtain that $\bar{u} \geq u$ in $B_{R}(\xi, R)$. As we have chosen $\xi \in \mathbb{R}$ arbitrarily we deduce that

$$
u\left(x_{1}, x_{2}\right)=0 \quad \text { for all } x_{1} \in \mathbb{R}, d(R) \leq x_{2} \leq 2 R-d(R) .
$$

Letting $R \rightarrow \infty$ and thanks to Lemma 4.3 we have that

$$
u\left(x_{1}, x_{2}\right)=0 \text { for all } x_{1} \in \mathbb{R}, x_{2} \geq c_{\nu q} .
$$

The boundedness for $x_{1}<0$ works similarly. Again we set

$$
\bar{u}\left(x_{1}, x_{2} ; \theta\right)=y_{R}\left(x_{1}-R \cos \theta, x_{2}-R \sin \theta\right) \quad \text { for } \pi / 2 \leq \theta \leq \pi,
$$

this time defined on $\Sigma_{\theta}=B_{R}(R \cos \theta, R \sin \theta) \cap \Omega$. The boundary of $\Sigma_{\theta}$ consists of the part of $\partial B_{R}(R \cos \theta, R \sin \theta)$ which is in $\Omega$ and where $\bar{u}=1$ and $u \leq 1$ and a part of $\partial \Omega$ with $x_{1}<0$ where $\bar{u} \geq 0$ and $u=0$. Once again, because we can move $\theta \in[\pi / 2, \pi]$, by the comparison principle we obtain that

$$
u\left(x_{1}, x_{2}\right)=0 \text { in }\left\{\left(x_{1}, x_{2}\right): x_{1} \leq 0, d(R)<r<2 R-d(R)\right\},
$$

with $r=\left(x_{1}^{2}+x_{2}^{2}\right)^{1 / 2}$. Letting $R \rightarrow \infty$,

$$
\left.u\left(x_{1}, x_{2}\right)=0 \quad \text { in }\left\{\left(x_{1}, x_{2}\right): x_{1} \leq 0, r \geq c_{\nu q}\right)\right\} .
$$

Remark 4.7. As in Remark 4.5 if in Lemma 4.6 we substitute $H_{v}$ with $\varepsilon H_{v}$, the result is the same but with $c_{\nu q}$ replaced by $c_{\nu q \varepsilon}$.

\subsection{Proof of Theorem 1.5}

For the proof of Theorem 1.5 we start by showing (ii) for $h=\varepsilon H_{v}$. We will use this result later to prove $(i i)$ for a general boundary datum. In a second moment we will show $(i)$ and give a numerical representation of the behaviour of $\bar{C}(q)$ and $\underline{C}(q)$ when $\varepsilon=1$.

\subsection{Heaviside function}

Problem (7) under assumption $H_{h p}$ and with $h=\varepsilon H_{v}$ is

$$
\begin{cases}-L u+u^{q}=0 & \text { in } \Omega, \\ u\left(x_{1}, 0\right)=\varepsilon H_{v}\left(x_{1}\right) & x_{1} \in \mathbb{R} .\end{cases}
$$


We remind that the symmetric matrix $A$

$$
A=\left(\begin{array}{ll}
a_{11} & a_{12} \\
a_{21} & a_{22}
\end{array}\right)
$$

satisfies

$$
\mu|\xi|^{2} \leq \xi^{T} A \xi \leq \nu|\xi|^{2}, \quad \text { for all } \xi \in \mathbb{R}^{2},
$$

for some $\mu, \nu>0$.

Remark 4.8. The existence of $\mu>0$ such that condition (54) holds is equivalent to the fact that

$$
a_{12}^{2}<a_{22} a_{11} .
$$

Indeed, if we set $\xi=\left(\xi_{1}, \xi_{2}\right)$, we have

$$
\begin{aligned}
\xi^{T} A \xi & =a_{11} \xi_{1}^{2}+2 a_{12} \xi_{1} \xi_{2}+a_{22} \xi_{2}^{2} \\
& =\mu\left(\xi_{1}^{2}+\xi_{2}^{2}\right)+\left(\left(a_{11}-\mu\right) \xi_{1}^{2}+2 a_{12} \xi_{1} \xi_{2}+\left(a_{22}-\mu\right) \xi_{2}^{2}\right) .
\end{aligned}
$$

In order for the second term to be greater or equal to zero for all $\xi_{1}, \xi_{2} \in \mathbb{R}$, it must hold

$$
a_{12}^{2}<\left(a_{11}-\mu\right)\left(a_{22}-\mu\right)<a_{11} a_{22} .
$$

This fact is used later on to obtain a subsolution.

In order to study the positivity set for the solution of $(53)$ we look for a proper subsolution. What we are really interested in is the behavior of the solution in a neighborhood of the origin, i.e., the point $(0,0)$. We will show that, although the boundary datum $\varepsilon H_{v}$ is zero for $x_{1}<0$, the solution is positive for $x_{1}>-\zeta_{\mu \varepsilon}$ and $x_{2}$ sufficiently small, for some $\zeta_{\mu \varepsilon}>0$.

The procedure to obtain a proper lower bound $\underline{u}$ for $\mathrm{u}$ is the same as the one in 20. We look for a $\underline{u}$ solution of

$$
\begin{cases}-L \underline{u}=-\varepsilon^{q} & \text { in } \mathbb{R} \times\left(0, c_{\nu q}\right), \\ \underline{u}\left(x_{1}, 0\right)=\varepsilon H\left(x_{1}\right) & x_{1} \in \mathbb{R} \\ \underline{u}\left(x_{1}, c_{\nu q \varepsilon}\right) \leq 0 & x_{1} \in \mathbb{R} .\end{cases}
$$

If such $\underline{u}$ exists, and remembering the bounds $0 \leq u \leq \varepsilon$ where $u$ is solution of (53), we get

$$
-L(\underline{u}-u)=-\varepsilon^{q}+u^{q} \leq 0 .
$$

This property, by the comparison principle, assures that $u \geq \underline{u}$.

To find out an explicit formula for $\underline{u}$, we split $\underline{u}=u_{1}+u_{2}$ with

$$
\left\{\begin{array}{cl}
-L u_{1}=-\varepsilon^{q} & x_{1} \in \mathbb{R}, 0<x_{2}<c_{\nu q \varepsilon}, \\
u_{1}\left(x_{1}, 0\right)=0, \quad u_{1}\left(x_{1}, c_{\nu q \varepsilon}\right)=-\varepsilon & x_{1} \in \mathbb{R},
\end{array}\right.
$$

and

$$
\left\{\begin{array}{lll}
-L u_{2}=0 & & x_{1} \in \mathbb{R}, 0<x_{2}<c_{\nu q \varepsilon}, \\
u_{2}\left(x_{1}, 0\right)=\varepsilon H\left(x_{1}\right), & u_{2}\left(x_{1}, c_{\nu q \varepsilon}\right) \leq \varepsilon & x_{1} \in \mathbb{R} .
\end{array}\right.
$$


We try, as in 20, to find a $u_{2}$ which depends only on the ratio $m=x_{1} / x_{2}$. Calling $f$ such a function, we have

$$
\sum_{i, j=1}^{2} a_{i j} \frac{\partial^{2}}{\partial x_{i} \partial x_{j}} f\left(x_{1} / x_{2}\right)=\frac{1}{x_{2}^{2}}\left(\left(a_{22} m^{2}-2 a_{12} m+a_{11}\right) f^{\prime \prime}+2\left(a_{22} m-a_{12}\right) f^{\prime}\right) .
$$

Since $x_{2}>0$, we look for a solution of the differential equation

$$
\left(a_{22} m^{2}-2 a_{12} m+a_{11}\right) f^{\prime \prime}+2\left(a_{22} m-a_{12}\right) f^{\prime}=0,
$$

which we see to be equivalent to $g f^{\prime \prime}+g^{\prime} f^{\prime}=\left(g f^{\prime}\right)^{\prime}=0$, where $g(m)=$ $a_{22} m^{2}-2 a_{12} m+a_{11}$. We deduce that $f^{\prime}=C g^{-1}$, where $C$ is a constant. Hence $f$ is given by the indefinite integral

$$
f=C \int \frac{1}{a_{22} m^{2}-2 a_{12} m+a_{11}} .
$$

From (55) we know that $a_{12}^{2}<a_{11} a_{22}$, which implies that

$$
f(m)=\bar{C}+C \frac{1}{\left(a_{11} a_{12}-a_{12}^{2}\right)^{1 / 2}} \arctan \left(\frac{a_{22} m-a_{12}}{a_{11} a_{12}-a_{12}^{2}}\right) .
$$

We set

$$
u_{2}\left(x_{1}, x_{2}\right)=f_{\varepsilon}\left(x_{1} / x_{2}\right),
$$

where $f_{\varepsilon}$ is $f$ with the constant $\bar{C}=\bar{C}_{\varepsilon}$ and $C=C_{\varepsilon}$ chosen for the specific problem. Let us check the boundary conditions: we fix $x_{1} \neq 0$ and send $x_{2} \rightarrow 0$. If $x_{1}<0$, then $x_{1} / x_{2} \rightarrow-\infty$ and $f_{\varepsilon}\left(x_{1} / x_{2}\right) \rightarrow \bar{C}_{\varepsilon}-C_{\varepsilon} \frac{\pi}{2}\left(a_{11} a_{12}-a_{12}^{2}\right)^{-1 / 2}$. Setting $C_{\varepsilon}=\varepsilon \pi^{-1}\left(a_{11} a_{12}-a_{12}^{2}\right)^{1 / 2}$ and $\bar{C}_{\varepsilon}=\varepsilon / 2$ we have that $f_{\varepsilon}\left(x_{1} / x_{2}\right) \rightarrow$ 0 . For $x_{1}>0$, we see that $f_{\varepsilon}\left(x_{1} / x_{2}\right) \rightarrow \varepsilon$. Since, with these values for the constants, $0 \leq f_{\varepsilon} \leq \varepsilon$, also the other boundary condition is satisfied.

For $u_{1}$ we choose

$$
u_{1}\left(x_{1}, x_{2}\right)=-\left(\frac{\varepsilon}{c_{\nu q \varepsilon}}+\frac{\varepsilon^{q} c_{\nu q \varepsilon}}{2}\right) x_{2}+\frac{\varepsilon^{q}}{2} x_{2}^{2} .
$$

It is immediate to verify that $u_{1}$ given by $(59)$ is solution of (57).

Now we check that $\underline{u}$ is positive in a neighbourhood of the origin. We know that, for $x_{2}=0$ and $x_{1}<0, \underline{u}$ is zero. We want to understand if it is positive for some $x_{2}>0$. We compute

$$
\frac{\partial \underline{u}}{\partial x_{2}}=-\left(\frac{\varepsilon}{c_{\nu q \varepsilon}}+\frac{c_{\nu q \varepsilon}}{2}\right)+x_{2}-\frac{C_{\varepsilon} x_{1}}{a_{22} x_{1}^{2}-a_{12} x_{1} x_{2}+a_{11} x_{2}^{2}},
$$

and evaluate it in $x_{2}=0$,

$$
\frac{\partial \underline{u}}{\partial x_{2}}\left(x_{1}, 0\right)=-\left(\frac{\varepsilon}{c_{\nu q \varepsilon}}+\frac{c_{\nu q \varepsilon}}{2}\right)-\frac{C_{\varepsilon}}{a_{22} x_{1}} .
$$

One can see that it is strictly positive for $x_{1}<0$ sufficiently close to zero, precisely in a neighbourhood of $\left(-\zeta_{\nu \varepsilon}, 0\right) \times\{0\}$, where

$$
\zeta_{\nu \varepsilon}=\frac{2 C_{\varepsilon} c_{\nu q \varepsilon}}{a_{22}\left(2 \varepsilon+c_{\nu q \varepsilon}^{2}\right)} \text {. }
$$




\subsection{General data}

In this part we show that the behaviour displayed by the solution of (53) can be found also in the solutions of (1) where $h$ is continuous, depending on the decay rate of $h$ near zero. If $h$ is sufficiently big when $x_{1} \sim 0$, then the free boundary is not connected with $\mathcal{S}(h)$.

We select the family of functions $\left\{h_{\delta}\right\} \subset C(\mathbb{R})$ of the form

$$
h_{\delta}\left(x_{1}\right)= \begin{cases}0 & x_{1} \leq 0 \\ C x^{\frac{2}{1-q}} & 0<x_{1} \leq x_{\delta} \\ 1 & x>x_{\delta}\end{cases}
$$

Proof of Theorem 1.5. For what concerns $(i)$, consider the solution $\underline{u}$ of with $\varepsilon=1$ and boundary data given by $H\left(x_{1}-x_{\delta}\right)$. It is immediate to check that $H\left(x_{1}-x_{\delta}\right) \leq h_{\delta}\left(x_{1}\right)$ for all $x_{1} \in \mathbb{R}$. This implies that $\underline{u} \leq u_{\delta}$ where $u_{\delta}$ is the solution of (53) with boundary data $h_{\delta}$. So if

$$
x_{\delta}=\left(\frac{1}{C}\right)^{\frac{1-q}{2}}
$$

is such that $x_{\delta}<\zeta_{\nu}$ then there exists $V$, neighbourhood of $(0,0)$ in $\mathbb{R}^{2}$, such that $\underline{u}>0$ in $V \cap\left\{x_{1}<0, x_{2}>0\right\}$. But this is true whenever

$$
C>\left(\frac{1}{\zeta_{\nu}}\right)^{\frac{2}{1-q}}
$$

It is enough to set $\bar{C}=\zeta_{\nu}^{-2 /(1-q)}, \bar{\varepsilon}=1$ and $\bar{x}_{1, \varepsilon}=(1 / \bar{C})^{(1-q) / 2}$. The statement follows since $\underline{u} \leq u_{\delta}$.

One can also try to repeat the same proof with $\varepsilon H\left(x_{1}-x_{\delta}\right)$ and compare the results to find the best lower bound for which the expansion on the boundary of the support property holds.

For the proof of $(i i)$ it is enough to consider the special case of $h_{\underline{\varepsilon}}\left(x_{1}\right)=$ $\underline{C} x_{1}^{\frac{2}{1-q}}$ for a.e. $x_{1} \in\left(0, \underline{x}_{1, \varepsilon}\right)$ and $h_{\underline{\varepsilon}}\left(x_{1}\right)=\underline{\varepsilon}$ for a.e. $x_{1} \in\left(\underline{x}_{1, \varepsilon},+\infty\right)$. Indeed, if $h \leq h_{\underline{\varepsilon}}$ then we know that the correspondent limit very weak solutions $u$ and $u_{\underline{\varepsilon}}$ satisfy that $0 \leq u \leq u_{\underline{\varepsilon}}$. So, if the non-diffusion of the boundary of the support property holds for $u_{\varepsilon}$ then it also holds for $u$.

Consider the function $\bar{u}=w\left(x_{1}+v x_{2}\right)$ where

$$
w(s)=C s_{+}^{\frac{2}{1-q}},
$$

with $C>0$. We compute

$$
\begin{aligned}
-L \bar{u}+\bar{u}^{q} & =-\left(a_{11}+2 a_{12} v+a_{22} v^{2}\right) w^{\prime \prime}+w^{q} \\
& =x_{+}^{2 /(1-q)}\left(-\left(a_{11}+2 a_{12} v+a_{22} v^{2}\right) \frac{2(1+q)}{(1-q)^{2}} C+C^{q}\right) .
\end{aligned}
$$

If we take

$$
C \leq \frac{(1-q)^{2}}{2(1+q)\left(a_{11}+2 a_{12} v+a_{22} v^{2}\right)},
$$

we have that $-L \bar{u}+\bar{u}^{q} \geq 0$, hence $\bar{u}$ is a supersolution. We notice that

$$
\bar{u}(\theta, r)=0 \quad \text { for } \theta \in \Theta=(-\pi / 2, \arctan (-v)) \text {, }
$$



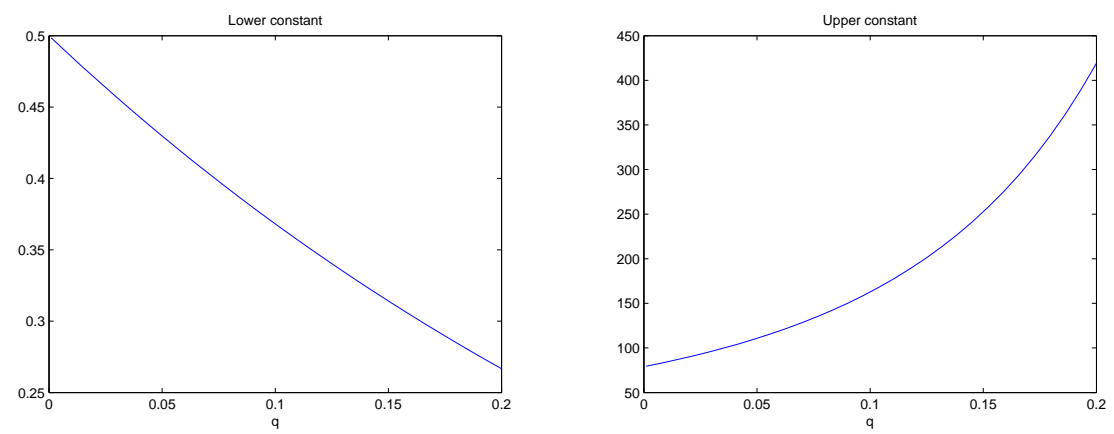

Figure 5: Graphs of $\underline{C}(q)$ and $\bar{C}(q)$

where $\theta=\arctan \left(x_{1} / x_{2}\right)$. Again, taking $\underline{C}$ smaller than 62 and choosing $\underline{x}_{1, \varepsilon}$ and $\underline{\varepsilon}$ such that $h_{\underline{\varepsilon}} \leq \bar{u}$, we have that $u \leq u_{\underline{\varepsilon}} \leq \bar{u}$; so we obtain that $u$ is zero in the sector $\Theta$.

Let us restrict our attention on the family of boundary data $h_{\delta}$. Each function is defined completely by the value of $C$, since $x_{\delta}$ can be deduced by $C$ and the continuity property. Of course for consistency we expect $\underline{C} \leq \bar{C}$. But can we affirm that equality holds? Actually, with our method we can not say. In fact, let us consider for example the case of $L=\Delta$, which means $A=I d$. We computed the values

$$
\bar{C}=\left(\frac{\pi\left(q^{2}-q+2\right)}{(1-q) \sqrt{2(q+1)}}\right)^{\frac{2}{1-q}} \quad \text { and } \quad \underline{C}=\frac{(1-q)^{2}}{2(1+q)} .
$$

We see from the graphs in Figure 5 that $\underline{C}<\bar{C}$ for $q \in(0,0.2)$, and the difference is quite big. For $q \in(0.2,1)$ the difference becomes even bigger as $C$ is decreasing while $\bar{C}$ is increasing. The question of what happen when $\underline{C}<\bar{C}<\bar{C}$ is still open and a different approach or a finer analysis is needed.

Proof of Corollary 1.6. The additional properties are a by-product of the proof of Theorem 1.5. Indeed, for (1), since the supersolution $w$ already satisfies this property, so does the solution because it is non-negative. Point (2) is true because we have shown that $u \geq u_{1}+u_{2}$ with $u_{1}$ and $u_{2}$ solutions of the problems (57) and (58) respectively and $\underline{u}=u_{1}+u_{2}$ satisfies the required properties.

\subsection{Proof of Corollary 1.13}

In the case of $(i)$ we can use the same supersolution (which we denote now by $\bar{u}(x))$ as for the stationary case. Since our initial condition is $u_{0}=0$, then, applying the comparison result, we get that $0 \leq u(t, x) \leq \bar{u}(x)$ for any $t>0$ and a.e. $\left(x_{1}, x_{2}\right) \in \mathbb{R} \times[0, \infty)$.

The proof of part (ii) comes from the fact that $u_{1}\left(x_{1}, x_{2}\right)+u_{2}\left(x_{1}, x_{2}\right)$ is a subsolution for the parabolic problem for $t \in(0, T]$. 


\section{References}

[1] R. A. Adams and J. J. F. Fournier. Sobolev spaces, volume 140 of Pure and Applied Mathematics (Amsterdam). Elsevier/Academic Press, Amsterdam, second edition, 2003.

[2] L. Alvarez and J. I. Díaz. On the retention of the interfaces in some elliptic and parabolic nonlinear problems. Discrete Contin. Dyn. Syst., 25(1):1-17, 2009.

[3] S. N. Antontsev, J. I. Díaz, and S. Shmarev. Energy methods for free boundary problems. Progress in Nonlinear Differential Equations and their Applications, 48. Birkhäuser Boston Inc., Boston, MA, 2002. Applications to nonlinear PDEs and fluid mechanics.

[4] D. E. Apushkinskaya, N. Matevosyan, and N. N. Uraltseva. The behavior of the free boundary close to a fixed boundary in a parabolic problem. Indiana Univ. Math. J., 58(2):583-604, 2009.

[5] D. E. Apushkinskaya and N. N. Uraltseva. Uniform estimates near the initial state for solutions of the two-phase parabolic problem. Algebra $i$ Analiz, 25(2):63-74, 2013.

[6] D. E. Apushkinskaya, N. N. Uraltseva, and Kh. Shakhgolyan. On global solutions of a parabolic problem with an obstacle. Algebra i Analiz, 14(1):325,2002 .

[7] R. Aris. The Mathematical Theory of Diffusion and Reaction in Permeable Catalysis. Clarendon Press, Oxford, 1975.

[8] P. Benilan, M. G. Crandall, and A. Pazy. Nonlinear Evolution Governed by Accretive Operators. Book to appear.

[9] Ph. Bénilan, M. G. Crandall, and P. Sacks. Some $L^{1}$ existence and dependence results for semilinear elliptic equations under nonlinear boundary conditions. Appl. Math. Optim., 17(3):203-224, 1988.

[10] Ph. Bénilan and P. Wittbold. Nonlinear evolution equations in Banach spaces: basic results and open problems. In Functional analysis (Essen, 1991), volume 150 of Lecture Notes in Pure and Appl. Math., pages 1-32. Dekker, New York, 1994.

[11] Ph. Benilan and P. Wittbold. On mild and weak solutions of ellipticparabolic problems. Adv. Differential Equations, 1(6):1053-1073, 1996.

[12] H. Brézis. Une équation semilinéaire avec conditions aux limites dans 11. 1972.

[13] H. Brézis. Opérateurs maximaux monotones et semi-groupes de contractions dans les espaces de Hilbert. North-Holland Publishing Co., Amsterdam, 1973. North-Holland Mathematics Studies, No. 5. Notas de Matemática (50).

[14] H. Brézis and W. A. Strauss. Semi-linear second-order elliptic equations in $L^{1}$. J. Math. Soc. Japan, 25:565-590, 1973. 
[15] M. G. Crandall and L. C. Evans. On the relation of the operator $\partial / \partial s+\partial / \partial \tau$ to evolution governed by accretive operators. Israel J. Math., 21(4):261-278, 1975.

[16] M. G. Crandall and A. Pazy. Semi-groups of nonlinear contractions and dissipative sets. J. Functional Analysis, 3:376-418, 1969.

[17] J. I. Díaz. Nonlinear partial differential equations and free boundaries. Vol. I, volume 106 of Research Notes in Mathematics. Pitman (Advanced Publishing Program), Boston, MA, 1985. Elliptic equations.

[18] J. I. Díaz and F. de Thélin. On a nonlinear parabolic problem arising in some models related to turbulent flows. SIAM J. Math. Anal., 25(4):10851111, 1994.

[19] J. I. Díaz and J.-M. Rakotoson. On the differentiability of very weak solutions with right-hand side data integrable with respect to the distance to the boundary. J. Funct. Anal., 257(3):807-831, 2009.

[20] C. J. van Duijn and L. A. Peletier. How the interface approaches the boundary in the dead core problem. J. Reine Angew. Math., 432:1-21, 1992.

[21] L. C. Evans. Nonlinear evolution equations in an arbitrary Banach space. Israel J. Math., 26(1):1-42, 1977.

[22] D. Kröner and J.-F. Rodrigues. Global behaviour for bounded solutions of a porous media equation of elliptic-parabolic type. J. Math. Pures Appl. (9), 64(2):105-120, 1985.

[23] J.-L. Lions and E. Magenes. Non-homogeneous boundary value problems and applications. Vol. I. Springer-Verlag, New York, 1972. Translated from the French by P. Kenneth, Die Grundlehren der mathematischen Wissenschaften, Band 181.

[24] M. Marcus and L. Véron. Initial trace of positive solutions of some nonlinear parabolic equations. Comm. Partial Differential Equations, 24(7-8):14451499, 1999.

[25] M. Marcus and L. Véron. Semilinear parabolic equations with measure boundary data and isolated singularities. J. Anal. Math., 85:245-290, 2001.

[26] M. Marcus and L. Véron. Nonlinear second order elliptic equations involving measures. De Gruyter Series in Nonlinear Analysis and Applications, Berlin, 2013.

[27] Y. Martel and P. Souplet. Small time boundary behavior of solutions of parabolic equations with noncompatible data. J. Math. Pures Appl. (9), 79(6):603-632, 2000.

[28] V. P. Mikhailov. Partial Differential Equations. Textbook. Mir. Moscú, 1978. 
[29] J.-F. Rodrigues. Obstacle problems in mathematical physics, volume 134 of North-Holland Mathematics Studies. North-Holland Publishing Co., Amsterdam, 1987. Notas de Matemática [Mathematical Notes], 114.

[30] H. Shahgholian and N. Uraltseva. Regularity properties of a free boundary near contact points with the fixed boundary. Duke Math. J., 116(1):1-34, 2003.

[31] J. L. Vázquez. The porous medium equation. Oxford Mathematical Monographs. The Clarendon Press Oxford University Press, Oxford, 2007. Mathematical theory.

[32] L. Véron. Elliptic equations involving measures. In Stationary partial differential equations. Vol. I, Handb. Differ. Equ., pages 593-712. NorthHolland, Amsterdam, 2004. 